\title{
Reasoning with Sets of Defaults in Default Logic
}

\author{
James P. Delgrande \\ School of Computing Science, \\ Simon Fraser University, \\ Burnaby, B.C., \\ Canada V5A 1S6, \\ jim@cs.sfu.ca
}

\author{
Torsten Schaub* \\ Institut für Informatik, \\ Universität Potsdam, \\ Postfach 6015 53, \\ D-14415 Potsdam, \\ Germany, \\ torsten@cs.uni-potsdam.de
}

March 9, 2004

\begin{abstract}
We present a general approach for representing and reasoning with sets of defaults in default logic, focussing on reasoning about preferences among sets of defaults. First, we consider how to control the application of a set of defaults so that either all apply (if possible) or none do (if not). From this, an approach to dealing with preferences among sets of default rules is developed. We begin with an ordered default theory, consisting of a standard default theory, but with possible preferences on sets of rules. This theory is transformed into a second, standard default theory wherein the preferences are respected. The approach differs from other work, in that we obtain standard default theories and do not rely on prioritised versions of default logic. In practical terms this means we can immediately use existing default logic theorem provers for an implementation. As well, we directly generate just those extensions containing the most preferred applied rules; in contrast, most previous approaches generate all extensions, then select the most preferred. In a major application of the approach, we show how semi-monotonic default theories can be encoded so that reasoning can be carried out at the object level. With this, we can reason about default extensions from within the framework of standard default logic. Hence one can encode notions such as skeptical and credulous conclusions, and can reason about such conclusions within a single extension.
\end{abstract}

Keywords: Knowledge representation, nonmonotonic reasoning, default logic, reasoning with preferences.

\section{Introduction}

In previous work [DS00a], we explored reasoning with preference among default rules in default logic [Rei80]. In that approach, one could state and reason about preference information, for

* Affiliated with Simon Fraser University, Burnaby, Canada. 
example that a car that is economical is preferred over one that is safe, and one that is safe is in turn preferred over one that is powerful. In the present paper, we extend this work to deal with preferences over sets of objects, represented by default rules. Thus, here one can state that a car that is safe and efficient is preferred over one that is cheap, efficient, and stylish.

We begin by showing how one can reason with sets of defaults in default logic. In particular we show how, for a set of defaults $D_{m}$, we can detect the case in which all defaults in $D_{m}$ apply. From this, for a default theory $\left(D \cup D_{m}, W\right)$ we show how to obtain a second theory wherein (informally) either all of the defaults in $D_{m}$ are applied (if possible) or none of them are. This is done by naming each of the defaults in $D_{m}$, and then expressing in default logic the applicability conditions for the defaults. These applicability conditions are then used to control the actual assertion of the default rule conclusions. We develop this in Section 4, following a background section and a section on motivation and intuitions.

Next, in Section 5 we develop a general methodology for reasoning with preferences among sets of defaults. We begin with an ordered default theory, a default theory with explicit preferences given on sets of default rules. This theory is translated into a standard default theory, where provably defaults are applied in the appropriate fashion. Roughly, we provide an axiomatisation that "considers" sets of defaults according to the given ordering. We employ a strongly prescriptive approach to dealing with preferences: informally, in building an extension, the maximum set(s) of default rules in the preference ordering are first considered, followed by the next-to-maximum, and so on. In particular, we forbid lower ranked defaults from enabling the activation of higher-ranked defaults, as is found in more descriptive approaches.

Last, in an application of the (general) approach, we show how the methodology for reasoning with sets can be modified so that for a large class of default theories, we can encode the set of extensions within a single extension. That is, in (standard) default logic, one typically obtains not just a single set of default conclusions, but rather multiple sets of candidate default conclusions; reasoning about these extensions is carried out at the meta-level. In Section 6, we show how such reasoning can be carried out at the object level. For a semi-monotonic ${ }^{1}$ default theory $\Delta=(D, W)$, we translate $\Delta$ to obtain a second theory $\Delta^{\prime}=\left(D^{\prime}, W^{\prime}\right)$, such that $\Delta^{\prime}$ has a single extension that encodes every extension of $\Delta$. Given this, one can express in the theory what it means for something to be a skeptical or credulous default conclusion. Hence one can reason about (skeptical and credulous) conclusions within the framework of a single extension of a default theory. The translation has several desirable properties. The translated theory $\Delta^{\prime}$ is only a constant factor larger than the original $\Delta$, with the exception of introduced unique names axioms. As well, here as in the previous sections, we prove that our translation behaves correctly. Only an outline of the application is described here; see [DS02] for the full development.

While there is a significant body of work treating preferences among individual properties in nonmonotonic approaches (see Section 2.2) this is, to the best of our knowledge, the first time that groups or sets of preferences have been so addressed. We adopt a methodology in which we remain within the framework of standard default logic, rather than building a scheme on top of default logic, as is found in other approaches. This methodology has several advantages. First, our approach can be immediately implemented by making use of an existing default logic theorem prover such as DeRes [CMT96]. As well, the approach allows the direct generation of extensions containing the most preferred (sets of) applied rules. This is in contrast with most previous work,

\footnotetext{
${ }^{1}$ This and other terms are defined in Section 2.
} 
in which extensions are generated and the most preferred subsequently selected. Third, it is easier to compare differing types of preference within a single framework. Moreover, in "compiling" sets and preferences into default logic, and in using the standard machinery of default logic, we obtain insight into the notion of preference orderings and reasoning with sets. Thus for example we implicitly show that explicit priorities over sets provide no real increase in the expressibility of default logic.

\section{Background}

\subsection{Default Logic}

We assume a basic familiarity with default logic [Rei80]; the following provides a précis of the approach. Default logic augments classical logic by default rules of the form $\frac{\alpha: \beta}{\gamma}$. A default rule is normal if $\beta$ is equivalent to $\gamma$; it is semi-normal if $\beta$ implies $\gamma$. We sometimes denote the prerequisite $\alpha$ of a default $\delta$ by $\operatorname{PRE}(\delta)$, its justification $\beta$ by $J U S(\delta)$, and its consequent $\gamma$ by $\operatorname{CON}(\delta)$. Accordingly, $\operatorname{PRE}(D)$ is the set of prerequisites of all defaults in $D ; J U S(D)$ and $\operatorname{CON}(D)$ are defined analogously. Empty components, such as no prerequisite or even no justifications, are assumed to be tautological. Semantically, defaults with unbound variables are taken to stand for all corresponding instances. A set of default rules $D$ and a set of formulas $W$ form a default theory $(D, W)$ that may induce zero, one, or multiple extensions in the following way [Rei80].

Definition 2.1 Let $(D, W)$ be a default theory and let $E$ be a set offormulas. Define $E_{0}=W$ and for $i \geq 0$ :

$$
\begin{aligned}
G D_{i} & =\left\{\frac{\alpha: \beta_{1}, \ldots, \beta_{n}}{\gamma} \in D \mid \alpha \in E_{i}, \neg \beta_{1} \notin E, \ldots, \neg \beta_{n} \notin E\right\} \\
E_{i+1} & =\operatorname{Th}\left(E_{i}\right) \cup\left\{\operatorname{CON}(\delta) \mid \delta \in G D_{i}\right\}
\end{aligned}
$$

Then $E$ is an extension for $(D, W)$ if $E=\bigcup_{i=0}^{\infty} E_{i}$.

Any such extension represents a possible set of beliefs about the world at hand. Further, define for a set of formulas $S$ and a set of defaults $D$, the set of generating default rules as $G D(D, S)=$ $\{\delta \in D \mid \operatorname{PRE}(\delta) \in S$ and $\neg J U S(\delta) \notin S\}$. An enumeration $\left\langle\delta_{i}\right\rangle_{i \in I}$ of default rules is grounded in a set of formulas $W$, if we have for every $i \in I$ that $W \cup \operatorname{CON}\left(\left\{\delta_{0}, \ldots, \delta_{i-1}\right\}\right) \vdash \operatorname{PRE}\left(\delta_{i}\right)$. A default theory $(D, W)$ is said to be semi-monotonic if, for $D^{\prime} \subseteq D^{\prime \prime} \subseteq D$, if $E^{\prime}$ is an extension of $D^{\prime}$ then there is an extension $E^{\prime \prime}$ of $D^{\prime \prime}$ where $E^{\prime} \subseteq E^{\prime \prime}$.

\subsection{Ordered Default Theories}

In [DS00a], we addressed the problem of reasoning about preferences among properties using default logic. This is part of a relatively large body of recent work dealing with preferences in nonmonotonic reasoning. Representative work in default logic includes [BH93, Bre94a, Rin95, BE00]. A distinguishing characteristic of [DSO0a] is that it translates ordered default theories into standard default theories, and doesn't necessitate extending the machinery of default logic. (However, see [GS97] for a related approach.) Preferences are first expressed in [DS00a] using 
an ordered default theory, $(D, W,<)$, where $D$ is a finite set of default rules, $W$ a finite set of formulas, and $<\subseteq D \times D$ a strict partial order on the default rules. That is, $<$ is a binary irreflexive and transitive relation on $D$. An ordered default theory is subsequently translated into a second, standard default theory wherein the preferences are respected, in that defaults are applied in the prescribed order. This translation is accomplished via the naming of defaults, so that reference may be made to a default rule from within a theory. Since the ordering $<$ is external to the theory $(D, W)$ such a theory is said to have static preferences. In this paper we will just deal with theories having static preferences.

For example, consider where one prefers a car that is not expensive $(E)$ over one with good safety features $(S)$ over one with a lot of power $(P)$. This might be expressed as $\frac{: P}{P}<\frac{: S}{S}<\frac{: E}{E} .^{2}$ Note that there is no syntactic relation between defaults in a preference relation. This is in contrast with ordered defaults employed for inheritance of properties, where the prerequisite of a more preferred default is less specific than a less preferred default; see [DS00b].

From this, the approach is generalised to theories with dynamic preferences, where preference information is specified within a default theory. Thus a theory is of the form $(D, W)$ where the relation < (effectively) can appear in elements of $D$ and $W$, as a binary relation in the object language on (names of) default rules. In this extension, one can specify preferences that hold by default, in a particular context, or give preferences among preferences.

\section{Reasoning with Sets of Defaults}

\subsection{Why Reason with Sets of Defaults?}

The discussion in the preceding subsection did not address the fact that some combinations of properties may outweigh others. In our previous car-buying example, one may also have the preference that safety features together with power is ranked over price. We consider therefore ordered default theories where the ordering is on sets of defaults. We can write our example as follows:

$$
\left\{\frac{: P}{P}\right\}<\left\{\frac{: S}{S}\right\}<\left\{\frac{: E}{E}\right\}<\left\{\frac{: P}{P}, \frac{: S}{S}\right\} .
$$

If we were given only that not all desiderata can be satisfied (and so $W=\{\neg(P \wedge S \wedge E)\}$ ) then intuitively we would want to apply the defaults in the set $\left\{\frac{: P}{P}, \frac{: S}{S}\right\}$ and conclude that $P$ and $S$ can be met. If instead we were given that $W=\{\neg P\}$ then the highest ranked set would be inapplicable, and we would want to apply the next ranked set, obtaining $E$, followed by the next ranked set, obtaining $S^{3}{ }^{3}$

Another application for preferences on sets of default rules comes from the domain of modelbased diagnosis [Rei87]. This approach appeals to the principle of parsimony, according to which a diagnosis is a conjecture that some minimal set of components is faulty. Often this is strengthened by preferring a single-fault diagnosis over a two-fault diagnosis, over a three-fault diagnosis, etc.

\footnotetext{
${ }^{2}$ See [BG94] for a more realistic, but analogous, encoding of such examples.

${ }^{3}$ This gives rise to the question of whether we want to consider all potentially applicable sets of defaults, in the order specified by $<$, or just the maximum applicable sets. As will become clear in Section 5, either possibility is easily representable.
} 
Assume one has three such components whose normal behaviour is modeled by means of rule $\frac{: \neg a b_{i}}{\neg a b_{i}}$ for $i=1 . .3$. We can model the strengthened principle of parsimony by

$$
\left\{\frac{: \neg a b_{i}}{\neg a b_{i}}\right\}<\left\{\frac{: \neg a b_{j}}{\neg a b_{j}}, \frac{: \neg a b_{k}}{\neg a b_{k}}\right\}<\left\{\frac{: \neg a b_{i}}{\neg a b_{i}}, \frac{: \neg a b_{j}}{\neg a b_{j}}, \frac{: \neg a b_{k}}{\neg a b_{k}}\right\}
$$

for $i, j, k=1 . .3$, where variables are local to a set and take distinct values.

Suppose our system description entails $a b_{1} \vee\left(a b_{2} \wedge a b_{3}\right)$. In standard default logic, we obtain two corresponding extensions, which would violate the strengthened principle of parsimony. With the given preferences, however, we obtain only the single-fault extension, containing $a b_{1}$ along with $\neg a b_{2}$ and $\neg a b_{3}$. In a similar fashion, in the realm of planning and in addressing the qualification problem, [Thi01] proposes sets of preferences over abnormalities.

For a somewhat different application involving sets of defaults, consider an issue identified and addressed by [BEFK99]. The claim made in that paper was that individuals that are indistinguishable in a (classical) theory should be treated uniformly by the set of defaults. In particular, we can consider the case where we have two defaults with conflicting consequents. Arguably all individuals who satisfy the prerequisite conditions for the (conflicting) defaults should be treated identically. The authors give the following example involving similar individuals:

- Students $(S)$ who have had bad grades in the past $(B)$ but unexpectedly obtain a good grade on an exam $(G)$ are normally suspected of cheating $(C)$.

- Students who are not caught cheating $(N)$ are normally not suspected of cheating.

This can be represented using the defaults

$$
D=\left\{\frac{S(x) \wedge B(x) \wedge G(x): C(x)}{C(x)}, \quad \frac{S(x) \wedge N(x): \neg C(x)}{\neg C(x)}\right\} .
$$

Assume further that Mary and John both are students who have had bad grades in the past, have received a good grade on an exam, and have not been caught cheating, viz:

$$
W=\{S(m), B(m), G(m), N(m), \quad S(j), B(j), G(j), N(j)\} .
$$

The theory $(D, W)$ has four extensions. Two extensions are arguably problematic, one containing $C(m), \neg C(j)$ and another containing $\neg C(m), C(j)$. Arguably, since John and Mary are indistinguishable according to the facts at hand, they should be treated the same that is to say, we should just obtain extensions with $C(m), C(j)$ or with $\neg C(m), \neg C(j)$. In this case we could group instances of the defaults, so that our theory would contain $\left\{\frac{S(m) \wedge B(m) \wedge G(m): C(m)}{C(m)}, \frac{S(j) \wedge B(j) \wedge G(j): C(j)}{C(j)}\right\}$ and $\left\{\frac{S(m) \wedge N(m): \neg C(m)}{\neg C(m)}, \frac{S(j) \wedge N(j): \neg C(j)}{\neg C(j)}\right\}$. Intuitively, we would want all defaults in a set to apply, or none. These sets would then be asserted to be preferred over their constituent elements.

\subsection{How to Reason with Sets of Defaults}

There are a number of issues that arise in considering sets of defaults and preferences among these sets. First, as described, we need some way of ensuring that all defaults in a set apply, or none do. Thus for the set $\left\{\frac{: P}{P}, \frac{R: S}{S}\right\}$, we do not want to apply the first default, unless the second default is 
applicable. This could happen if, for example, we had the default $\frac{: R}{R}$ elsewhere in the theory. It may also be that the defaults within a set depend on each other. So if we had the set $\left\{\frac{: P}{P}, \frac{P: S}{S}\right\}$, then the application of the first default would enable application of the second, thus enabling both defaults in the set to be applied.

There are also some subtleties as to how sets of defaults may interact once preferences are introduced. Consider the set-based default theory

$$
\left(\left\{\left\{\frac{: P}{P}, \frac{: R}{R}\right\},\left\{\frac{P: S}{S}, \frac{: T}{T}\right\}\right\}, \emptyset\right)
$$

The first set of defaults can be jointly applied. The second set cannot be applied in isolation; however once the first set of defaults has been applied, the conclusion of $P$ allows the second set to be jointly applied. Hence we would expect to obtain an extension containing $P, R, S$, and $T$.

Thus, if we added the preference

$$
\left\{\frac{P: S}{S}, \frac{: T}{T}\right\}<\left\{\frac{: P}{P}, \frac{: R}{R}\right\}
$$

then we would expect precisely the same outcome: the most preferred set is applicable and, once applied, the second set can be applied.

The situation is not so clear for the reverse preference:

$$
\left\{\frac{: P}{P}, \frac{: R}{R}\right\}<\left\{\frac{P: S}{S}, \frac{: T}{T}\right\} .
$$

One could argue that the less-preferred set is applicable, and that the application of this set would then enable the application of the more preferred set. Hence, so the argument might run, we should obtain an extension containing $P, R, S$, and $T$. In contrast to such a descriptive interpretation of preferences, one can take a a prescriptive approach, and assert that the <-greatest set should be determined to be applicable (or not), followed in order by less preferred sets. Under this interpretation, one might expect to obtain an extension containing $P$ and $R$ only.

Here (as in [DS00a]) we adopt a prescriptive approach. First, there is reason to believe that a prescriptive approach will generally be more efficient than a descriptive approach (even though the respective complexity classes may be the same). Second, it seems that a descriptive approach requires (at least in its obvious implementation) a meta-level approach, whereby extensions are generated and filtered according to the preference criteria. As we have noted, a prescriptive approach allows preferred extensions to be generated directly. Lastly, a prescriptive interpretation arguably comes with more representational force and allows a tighter characterisation of a domain. That is, a prescriptive approach forces a knowledge base designer to be explicit about what things should be applied in what order. For the example (3), if the more preferred set is intended to be applied in light of the applicability of the less preferred set, then this should be explicitly encoded in the domain theory, something that a prescriptive interpretation requires. A descriptive interpretation on the other hand simply gives a "wish list" of preferences which may or may not be meaningful. See [DSO0b] for more details.

\subsection{The Approach}

The general approach is similar to that in [DS00a, DS00b]; the present paper can be seen as an extension of the methodology proposed and developed in this previous work. Essentially, we 
have proposed a general methodology for using default logic, involving the naming of default rules and the introduction of special-purpose predicates, for detecting default rule applicability and controlling a rule's application. This allows the encoding of specific strategies and policies governing the set of default rules.

The general framework is quite straightforward. We begin with a language for expressing some phenomenon in which we are interested, along with an informal notion of what inferences should obtain in this language. For the present paper, we deal with set-ordered default theories, consisting of a default theory along with possible preferences on subsets of the defaults. A translation is given, such that the original theory is mapped into a (standard, Reiter) default theory, wherein the obtained extensions contain just the applicable, preferred (sets of) conclusions. The translation takes a default theory, augmented by a preference relation among sets of defaults, and maps this onto a standard, Reiter default theory, where mention of sets and explicit preferences is eliminated and replaced by special-purpose tags (predicates) that play the same role as the grouping of defaults into sets.

This translation serves several purposes. In a sense it provides a semantics for (or formalises) the original theory, in that it demonstrates how the original theory is expressible in default logic. That is, the desiderata sketched in the previous subsection are quite informal; part of the intent of the approach then is to formalise these intuitions. In particular, we are able to prove that our approach has the desired properties. Second, the translation provides a direction for implementation: given an efficient translation into default logic and a default logic theorem prove (e.g. [CMT96]), it is straightforward to implement the desired inferences of the original theory.

To illustrate the general idea of our methodology, consider a default rule $\frac{\alpha: \beta}{\gamma}$. Assume that constant $n$ is associated with this rule as its name in the object theory. Then the rule $\frac{\neg \beta \text { : }}{\mathrm{bl}(n)}$ will be applied just when the original rule cannot be applied due to its justification being not consistent with the set of beliefs. $\mathrm{bl}(\cdot)$ is a newly-introduced predicate; the concluding of $\mathrm{bl}(n)$ signals in the object theory that the original rule is not applied. Similarly, if we replace the rule $\frac{\alpha: \beta}{\gamma}$ by $\frac{\text { ok }(n) \wedge \alpha: \beta}{\gamma}$, where $\mathrm{ok}(\cdot)$ is a newly-introduced predicate, then application of this rule can be controlled in the object theory, in that the rule cannot be applied unless ok $(n)$ has been asserted. This notion of adding "tags" to detect and control rule application yields surprisingly powerful results. As described, we have shown in [DS00a] how an ordered default theory, consisting of a theory with a partial order on the default rules, can be translated into a "standard" default theory in which the rule ordering is adhered to.

In order to be able to reason with preferences among sets of defaults, there are two issues that need to be addressed. The first concerns being able to "control" an individual set of rules so that all of the defaults in the set apply, or none do. This is covered as a separate topic in the next section. Second, once we can deal with a set of defaults in isolation, this is extended to reasoning with other sets of defaults where there may be a preference ordering on these sets. This is the subject of Section 5. There we modify the techniques used in [DSO0a], so as to be able to deal with sets of defaults. Lastly, we describe an application of the approach, that of encoding semi-monotonic default theories, discussed in the next subsection and developed formally in Section 6. 


\subsection{Encoding (Some) Default Theories in Default Logic}

In nonmonotonic reasoning, in so-called consistency-based approaches such as default logic [Rei80] and autoepistemic logic [Moo85], one typically obtains not just a single set of default conclusions, but rather multiple sets of candidate default conclusions. Consider the (hackneyed) example wherein Quakers are normally pacifist, republicans are normally not, and where adults are normally employed. Assume as well that someone is a Quaker, republican, and an adult. In default logic this can be encoded by: $\left(\left\{\frac{Q: P}{P}, \frac{R: \neg P}{\neg P}, \frac{A: E}{E}\right\},\{Q, R, A\}\right)$. This theory has two extensions or sets of default conclusions, one containing $\{Q, R, A, E, P\}$ and the other $\{Q, R, A, E, \neg P\}$. In autoepistemic logic the same example appropriately encoded yields two analogous expansions or possible belief sets.

Reasoning about these extensions (resp. expansions) is carried out at the meta-level: a default conclusion that appears in some extension is called a credulous (or brave) default conclusion, while one that appears in every extension is called a skeptical conclusion. In the preceding example, $P$ is a credulous default conclusion, while $E$ is a skeptical conclusion. Intuitively it might seem that skeptical inference is the more useful notion. However, this is not necessarily the case. In diagnosis from first principles [Rei87] for example, in one encoding there is a 1-1 correspondence between diagnoses and extensions of the (encoding) normal default theory. Hence one may want to carry out further reasoning to determine which diagnosis to pursue. More generally there may be reasons to somehow synthesise the information found in several extensions.

In Section 6, we show how such reasoning can be carried out at the object level. For a default theory $\Delta=(D, W)$, we translate $\Delta$, by adapting the methodology of Section 5 , to obtain a second theory $\Delta^{\prime}=\left(D^{\prime}, W^{\prime}\right)$, such that $\Delta^{\prime}$ has a single extension that encodes every extension of $\Delta$. Given this, one can express in the theory what it means for something to be a skeptical or credulous default conclusion. This result isn't completely general; however it applies to semimonotonic default theories.

So in this approach we can encode the notion of "extension" within the framework of standard default logic. Hence one can reason about (skeptical and credulous) conclusions within the framework of a single extension of a default theory. Thus for example, in a diagnosis setting one could go on and axiomatise notions of preference among diagnoses having to do with, perhaps, number of faulty components, or based on components expected to fail first. This result has some theoretical interest, in that it shows (for theories that we consider) how multiple extensions are encodable in a single extension, with no significant overhead.

\section{Applying All, or None, of a Set of Defaults}

In this section we consider the problem of how to uniformly apply all defaults in some set. That is, if all defaults in the set can be simultaneously applied, then we want to apply all the defaults; otherwise (if some default cannot be applied in the context of the other defaults), we wish no default in the set to be applied. We will work with default theories $(D, W)$ having some distinguished finite subset $D_{m} \subseteq D$. For making the set $D_{m}$ explicit, we denote such theories by $\left(D \cup D_{m}, W\right)$. The idea is that we wish to obtain extensions of $\left(D \cup D_{m}, W\right)$ subject to the constraint that all defaults in $D_{m}$ are applied, or none are. For example, in the theory $\left(\left\{\frac{: A}{A}\right\} \cup\left\{\frac{: B}{B}, \frac{C: D}{D_{C}}\right\}, \emptyset\right)$ we would want to obtain an extension containing $A$, but not $B$ (since the defaults in $\left\{\frac{: B}{B}, \frac{C: D}{D}\right\}$ cannot both 
be applied). For $\left(\left\{\frac{: A}{A}\right\} \cup\left\{\frac{: B}{B}, \frac{B: D}{D}\right\}, \emptyset\right)$ we would want to obtain an extension containing $A, B$, and $D$.

Each default is associated with a unique name. This is done by extending the original language by a set of constants ${ }^{4} N$ such that there is a bijective mapping $n: D \rightarrow N$. We write $n_{\delta}$ instead of $n(\delta)$ (and we often abbreviate $n_{\delta_{i}}$ by $n_{i}$ to ease notation). Also, for default $\delta$ along with its name $n$, we sometimes write $n: \delta$ to render naming explicit. To encode the fact that we deal with a finite set of distinct default rules, we adopt a unique names assertion ( $\left.\mathrm{UNA}_{N}\right)$ and domain closure assertion $\left(\mathrm{DCA}_{N}\right)$ with respect to $N$. So, for a name set $N=\left\{n_{1}, \ldots, n_{k}\right\}$, we add axioms

$$
\begin{aligned}
& \mathrm{UNA}_{N}: \neg\left(n_{i}=n_{j}\right) \text { for all } n_{i}, n_{j} \in N \text { with } i \neq j \\
& \operatorname{DCA}_{N}: \forall x . \operatorname{name}(x) \equiv\left(x=n_{1} \vee \ldots \vee x=n_{k}\right) .
\end{aligned}
$$

We write $\forall x \in N . P(x)$ for $\forall x$. name $(x) \supset P(x)$.

Further, we introduce a new constant $m$ as the name of the designated rule set $D_{m}$. The name, $m$, of this rule set is related with the names of its members by a new binary predicate in where in $(x, y)$ is true just if the default named by $x$ is a member of the set named by $y$. In this section, instances of $i n$ will be of the form $i n(\cdot, m)$. While we could get away with not using in (and $m$ ) here, this additional machinery is required in Sections 5 and 6, and it is most straightforward to introduce it here. Note that we do not need a full axiomatisation of $i n$, representing set membership, since we use it in a very restricted fashion.

For applying all, or none, of the defaults in $D_{m}$, we need to be able to, first, detect when a rule is blocked and, second, control the application of a rule based on other prerequisite conditions. There are two cases for a default $\frac{\alpha: \beta}{\gamma}$ to be blocked: the prerequisite is not known to be true (and so its negation $\neg \alpha$ is consistent), or the justification is not consistent (and so its negation $\neg \beta$ is derivable). For detecting these cases, we introduce a new, special-purpose predicate bl(.). For controlling application of a rule we introduce predicates ok $(\cdot)$ and $\mathrm{ko}(\cdot)$. Similarly we introduce a specialpurpose predicate $\operatorname{ap}(\cdot)$ to detect when a rule has been applied. This last predicate isn't required here, but it is needed in later sections; as well it allows a compact and transparent statement of results in Theorem 4.1.

Definition 4.1 Given a default theory $\left(D \cup D_{m}, W\right)$ over language $\mathcal{L}$ and its set of associated default names $N \cup \dot{\cup}\{m\},{ }^{5}$ define $\mathcal{S}_{m}\left(\left(D \cup D_{m}, W\right)\right)=\left(D^{\prime}, W^{\prime}\right)$ over $\mathcal{L}^{*}$ by

$$
\begin{aligned}
D^{\prime} & =D \cup D_{N} \cup D_{M} \\
W^{\prime} & =W \cup W_{D} \cup W_{M} \cup\left\{D C A_{N}, U N A_{N}\right\}
\end{aligned}
$$

where

$$
\begin{aligned}
D_{N} & =\left\{\frac{\alpha \wedge \mathrm{ok}(n): \beta}{\gamma \wedge \mathrm{ap}(n)} \mid n: \frac{\alpha: \beta}{\gamma} \in D_{m}\right\} \\
D_{M} & =\left\{\frac{: \neg \mathrm{ko}(m)}{\mathrm{ok}\left(n_{1}\right) \wedge \ldots \wedge \mathrm{ok}\left(n_{k}\right)} \mid n_{i}: \frac{\alpha_{i}: \beta_{i}}{\gamma_{i}} \in D_{m}, i=1 . . k\right\} \\
& \cup\left\{\frac{: \neg \alpha}{\mathrm{bl}(m)}, \frac{([\forall y \in N . \operatorname{in}(y, m) \supset c(y, m)]) \supset \neg \beta:}{\mathrm{b} \mid(m)} \mid n: \frac{\alpha: \beta}{\gamma} \in D_{m},\right\} \\
W_{D} & =\left\{c\left(n_{\delta}, m\right) \equiv \operatorname{CON}(\delta) \mid \delta \in D_{m}\right\}
\end{aligned}
$$

\footnotetext{
${ }^{4}$ [McC86] first suggested naming defaults using a set of aspect functions. See also [Poo88, Bre94b].
}

${ }^{5}$ We let $\cup \dot{~ s t a n d ~ f o r ~ d i s j o i n t ~ u n i o n . ~}$ 


$$
\begin{aligned}
W_{M} & =\left\{\forall x \in N \cdot i n(x, m) \equiv\left(x=n_{1} \vee \ldots \vee x=n_{k}\right)\right\} \\
& \cup\{\mathrm{bl}(m) \supset \operatorname{ko}(m)\} \\
& \cup\{(\forall x \in N \cdot \operatorname{in}(x, m) \supset \operatorname{ap}(x)) \supset \operatorname{ap}(m)\}
\end{aligned}
$$

$D_{N}$ contains the images of the original rules in $D_{m}$. Each rule $\delta_{j} \in D_{N}$ is applicable, if ok $\left(n_{j}\right)$ is derivable. In fact, we assert ok $\left(n_{j}\right)$ for every $\delta_{j} \in D_{m}$, unless we cannot jointly apply all rules of $D_{m}$. That is, before activating the constituent rules, we have to make sure that none of them will be blocked. This is accomplished through the justification $\neg \mathrm{ko}(m)$ in (5) together with Axiom (9). We block the rule in (5) (and with it the derivability of all ok $\left(n_{j}\right)$ ) when we detect that one of $\delta_{1}, \ldots, \delta_{k}$ is blocked (since $\mathrm{ko}(m)$ is an immediate consequence of $\mathrm{bl}(m)$ ).

Now, we have that $D_{m}$ is blocked $(\mathrm{bl}(m))$ just if some rule in $D_{m}$ is blocked. However, since we must control a set of defaults, we must check for the blocking of one of the constituent default rules in the context of all other rules in the set applying. This motivates the prerequisite of the second rule in (6). First, we express the consequents of the rules in $D_{m}$ by means of a function (see (7)); this avoids a quadratic blowup in space in our translated theory. ${ }^{6}$ This context, effectively $\left(\gamma_{1} \wedge \ldots \wedge \gamma_{k}\right)$, is not needed for detecting the failure of derivability via the first rule in (6), since this test is with respect to the final extension $E$ via $\neg \alpha_{j} \notin E$.

This also brings up the relationship between $\mathrm{bl}(m)$ and $\mathrm{ko}(m)$. ko is used generally to eliminate a set of rules from consideration. In this and the next section, ko of a set is asserted precisely when bl is asserted - i.e. a set is flagged as ko'ed just when it is known that all defaults in the set cannot be jointly applied. However in Section 6 there will be other reasons to eliminate a set of rules from consideration. Consequently we have separate predicates to indicate that a set is bl'ed, and the more general ko.

Finally, as given in (10), $D_{m}$ is applied $(\operatorname{ap}(m))$ just if every rule in $D_{m}$ is applied; it is only in this last case that the consequents of the constituent rules in $D_{m}$ are asserted. (Note that the consequents of the constituent rules may be in an extension, even if ap $(m)$ hasn't been asserted, for example if the consequents were originally in the set $W$.)

Consider theory $\left(D \cup D_{m}, W\right)$, where

$$
D=\left\{\frac{: E}{E}\right\}, D_{m}=\left\{n_{1}: \frac{: P}{P}, n_{2}: \frac{: S}{S}\right\} .
$$

For $D_{N}$ and $D_{M}$, we obtain (after simplifying and removing redundant defaults):

$$
\frac{\mathrm{ok}\left(n_{1}\right): P}{P \wedge \mathrm{ap}\left(n_{1}\right)}, \quad \frac{\mathrm{ok}\left(n_{2}\right): S}{S \wedge \mathrm{ap}\left(n_{2}\right)}, \quad \frac{: \neg \mathrm{ko}(m)}{\mathrm{ok}\left(n_{1}\right) \wedge \mathrm{ok}\left(n_{2}\right)}, \quad \frac{\neg P \vee \neg S:}{\mathrm{bl}(m)} .
$$

The in predicate has instances: in $\left(n_{1}, m\right)$ and $i n\left(n_{2}, m\right)$.

Let $W=\{\neg(P \wedge E \wedge S)\}$. We obtain two extensions, one containing $P, S, \neg E$ and the other containing $E, \neg(P \wedge S)$. For the first extension, we obtain ok $\left(n_{1}\right)$ and ok $\left(n_{2}\right)$. If both $\delta_{1}$ and $\delta_{2}$ are applicable (which they are) then we conclude $P$ and $S$ and so $\neg E$. For the other extension, if the default $\frac{: E}{E}$ is applied, then $\neg P \vee \neg S$ is derivable, and so $\frac{\neg P \vee \neg S:}{\mathrm{bl}(m)}$ is applicable, from which we obtain $\mathrm{bl}(m)$, and so $\mathrm{ko}(m)$, blocking application of $\frac{: \neg \mathrm{ko}(m)}{\mathrm{ok}\left(n_{1}\right) \wedge \mathrm{ok}\left(n_{2}\right)}$. Consequently neither $\frac{\mathrm{ok}\left(n_{1}\right): P}{P \wedge \operatorname{ap}\left(n_{1}\right)}$ nor $\frac{\text { ok }\left(n_{2}\right): S}{S \wedge \text { ap }\left(n_{2}\right)}$ can be applied.

\footnotetext{
${ }^{6}$ As well, this anticipates the more intricate encodings in the next two sections.
} 
In the next example, defaults inside a set depend upon each other. Consider $\left(\emptyset \cup D_{m}, \emptyset\right)$ with

$$
D_{m}=\left\{n_{1}: \frac{: Q}{Q}, n_{2}: \frac{Q: R}{R}\right\} .
$$

We get for $D_{N}$ and $D_{M}$, after simplifying, the following rules.

$$
\frac{\mathrm{ok}\left(n_{1}\right): Q}{Q \wedge \mathrm{ap}\left(n_{1}\right)}, \quad \frac{Q \wedge \mathrm{ok}\left(n_{2}\right): R}{R \wedge \mathrm{ap}\left(n_{2}\right)}, \quad \frac{: \neg \mathrm{ko}(m)}{\mathrm{ok}\left(n_{1}\right) \wedge \mathrm{ok}\left(n_{2}\right)}, \quad \frac{\neg Q \vee \neg R:}{\mathrm{bl}(m)}, \quad \frac{: \neg Q}{\mathrm{bl}(m)} .
$$

We obtain ok $\left(n_{1}\right)$, and ok $\left(n_{2}\right)$, which allow us to apply default $\delta_{1}$, yielding in turn $Q \wedge \operatorname{ap}\left(n_{1}\right)$. Given $Q$, we can now apply default $\delta_{2}$, yielding $R \wedge$ ap $\left(n_{2}\right)$. We thus get an extension containing $Q$ and $R$. This example shows why we cannot avoid the translation by replacing $D_{m}$ by $\frac{\bigwedge_{\delta \in D_{m}} \operatorname{PRE}(\delta): \bigwedge_{\delta \in D_{m}} J U S(\delta)}{\bigwedge_{\delta \in D_{m}} \operatorname{CON}(\delta)}$.

The next theorem summarises properties of our approach, and shows that the rules in $D_{m}$ are applied either en masse, or not at all.

Theorem 4.1 Let $E$ be a consistent extension of $\mathcal{S}_{m}\left(\left(D \cup D_{m}, W\right)\right)$ for default theory $(D \cup$ $\left.D_{m}, W\right)$. We have that:

1. $\operatorname{ap}(m) \in E$ iff $\left\{\operatorname{ap}\left(n_{\delta}\right) \mid \delta \in D_{m}\right\} \cup \operatorname{CON}\left(D_{m}\right) \subseteq E$

2. $\mathrm{bl}(m) \in E$ iff $\left\{\operatorname{ap}\left(n_{\delta}\right) \mid \delta \in D_{m}\right\} \nsubseteq E$

3. $\operatorname{ok}\left(n_{\delta}\right) \in E$ iff $\operatorname{ap}\left(n_{\delta}\right) \in E$

4. ok $\left(n_{\delta}\right) \in E$ for all $\delta \in D_{m}$ iff $\mathrm{ko}(m) \notin E$

5. $\operatorname{ap}\left(n_{\delta}\right) \in E$ for $\delta \in D_{m}$ iff $\left\{\operatorname{ap}\left(n_{\delta}\right) \mid \delta \in D_{m}\right\} \subseteq E$.

The first part of the theorem states that the set is applied exactly when every rule in the set is applied, while the second part states that the set is blocked exactly when some rule is not applied. From Part 3 of the theorem we get that a rule is flagged as ok precisely if it can be applied. The last two parts assert that all rules are flagged as ok precisely if the set isn't blocked, and if one rule is applied in $D_{m}$ then all rules are applied.

Theorem 4.2 For default theory $(\emptyset \cup D, W)$, we have that $\mathcal{S}_{m}((\emptyset \cup D, W))$ has extension $E$ where either $E \cap \mathcal{L}=\operatorname{Th}(W \cup \operatorname{CON}(D))$ or else $E \cap \mathcal{L}=\operatorname{Th}(W)$.

The above theorem implies that, regardless of the form of the defaults in our set, we are guaranteed that the theory has an extension. For example, the default theory $\left(\emptyset \cup\left\{\frac{: B}{\neg B}\right\}, \emptyset\right)$ has an extension $E$ where $E \cap \mathcal{L}=\operatorname{Th}(\emptyset)$.

Theorem 4.3 Let $(D, W)$ be a (standard) default theory over $\mathcal{L}$ with extension $E$ and (respective) set of generating defaults $G D(D, E)$. Then $\mathcal{S}_{m}((\emptyset \cup G D(D, E), W))$ has extension $E^{\prime}$ where $E=E^{\prime} \cap \mathcal{L}$. 


\section{Preferences on Sets of Defaults}

In this section we consider default theories in which there may be preferences among subsets of the defaults. Consider again example (1), where in buying a car one ranks the price $(E)$ over safety features $(S)$ over power $(P)$, but safety features together with power is ranked over price. Using named defaults and sets, we can write this as:

$$
m_{1}:\left\{\frac{: P}{P}\right\}<m_{2}:\left\{\frac{: S}{S}\right\}<m_{3}:\left\{\frac{: E}{E}\right\}<m_{1,2}:\left\{\frac{: P}{P}, \frac{: S}{S}\right\}
$$

where $m_{1}, m_{2}, m_{3}$, and $m_{1,2}$ are names of sets of defaults; we describe their use later. Intuitively, if we were given only that not all desiderata can be satisfied (i.e. $W=\{\neg(P \wedge E \wedge S)\}$ ) then we could apply the defaults in the set named $m_{1,2}$ and conclude that $P$ and $S$ can be met. This should be the only extension.

So now a default theory has an ordering given on sets of defaults. Consider a general assertion $D^{\prime}<D^{\prime \prime}$ where $D^{\prime}, D^{\prime \prime} \subseteq D$. Informally we prefer the application of the set $D^{\prime \prime}$ to that of $D^{\prime}$. We can say that $D^{\prime \prime}$ is applicable if all its member defaults are, and inapplicable if one of its members is inapplicable. Consequently we consider $D^{\prime}$ after all defaults in $D^{\prime \prime}$ are found to be applicable, or some default in $D^{\prime \prime}$ is found to be inapplicable.

Definition 5.1 A set-ordered default theory is a triple $(D, W,<)$, where $D$ is a finite set of default rules, $W$ is a finite set of formulas, and $<\subseteq 2^{D} \times 2^{D}$ is a strict partial order.

In order to refer to the sets involved in $<$, we define $\left.2^{D}\right|_{<}=\left\{D^{\prime}, D^{\prime \prime} \mid\left(D^{\prime}, D^{\prime \prime}\right) \in<\right\}$.

The notation of the previous section is extended as follows. Names are introduced for each subset of $D$, and for each instance of a rule in each subset of $D$. As well, new predicate symbols are introduced to further control the application of sets of rules. We then give a translation that yields a second default theory $\left(D^{\prime}, W^{\prime}\right)$. Viewed algorithmically, this second theory carries out the following: If a maximal set of defaults $D$ is applicable, then the default's consequents are asserted. These consequents are "propagated" to all sets that are not <-greater than the set at hand. Following this, the next $<$-maximal sets are considered, and the process continues through the partial order. Crucially, default conclusions are "tagged" with the name of the set in which they appear so as to eliminate possible side effects. However, at the same time, we want the consequences of one set to be available to another set, provided the second set is not $<$-greater than the first. Consider sets $m_{1}:\left\{\frac{: A}{A}\right\}$ and $m_{2}:\left\{\frac{A: B}{B}\right\}$ with no preference relation between them. The rules are mapped into set-indexed rules, say, for the purposes of illustration, ${ }^{7} \frac{: A\left(m_{1}\right)}{A\left(m_{1}\right)}$ and $\frac{A\left(m_{2}\right): B\left(m_{2}\right)}{B\left(m_{2}\right)}$. We can apply the default of the first set, obtaining $A\left(m_{1}\right)$. Since we do not have $m_{1}<m_{2}$ we also assert $A\left(m_{2}\right)$ allowing the second set to be applied.

To name sets of defaults, we take some fixed enumeration $\left\langle n_{1}, \ldots, n_{k}\right\rangle$ of $N$, and define $m$ as a $k$-ary function symbol. Then, for $n_{\perp} \notin N$ and $\vec{x}=x_{1} . . x_{k}$, define

$$
\operatorname{DCA}_{M}: \forall \vec{x} \text {. set-name }(m(\vec{x})) \equiv\left(x_{1}=n_{1} \vee x_{1}=n_{\perp}\right) \wedge \ldots \wedge\left(x_{k}=n_{k} \vee x_{k}=n_{\perp}\right) .
$$

Intuitively, $x_{i}=n_{\perp}$ tells us that $n_{i}$ does not belong to the set at hand.

\footnotetext{
${ }^{7}$ The actual mapping given in Definition 5.3 is more complex.
} 
Accordingly, for $\vec{x}=x_{1} \ldots x_{k}$ and $\vec{x}^{\prime}=x_{1}^{\prime} . . x_{k}^{\prime}$ define

$$
\mathrm{UNA}_{M}: \forall \vec{x}, \vec{x}^{\prime} . \text { set-name }(m(\vec{x}))=\operatorname{set-name}\left(m\left(\vec{x}^{\prime}\right)\right) \equiv x_{1}=x_{1}^{\prime} \wedge \ldots \wedge x_{k}=x_{k}^{\prime} .
$$

The advantage of this "vector-oriented" representation over a dynamic one including a binary function symbol (as with lists) is that each set has a unique representation. ${ }^{8}$ We write $\forall x \in M . P(x)$ instead of $\forall x$. set-name $(x) \supset P(x)$. Further, we use $M$ for denoting the set of all valid set-names, that is,

$$
M=\left\{m\left|\mathrm{DCA}_{M}\right|=\operatorname{set} \text {-name }(m)\right\} .
$$

In order to ease notation, we write $m_{1,3}$ as the name of the set $\left\{\delta_{1}, \delta_{3}\right\}$ instead of $m\left(n_{1}, n_{\perp}, n_{3}, n_{\perp}, \ldots, n_{\perp}\right)$. Also, we abbreviate $m\left(n_{\perp}, \ldots, n_{\perp}\right)$ by $m_{\emptyset}$ and $m\left(n_{1}, \ldots, n_{k}\right)$ by $m_{D}$. Note the difference between names $n_{i}$ and $m_{i}$, induced by our notational convention.

We now use the "vector-oriented" representation for capturing set membership, denoted by in $(\cdot, \cdot)$. Consider for instance $N=\left\{n_{1}, n_{2}\right\}$. Membership is axiomatised through the formulas

$$
\begin{aligned}
& \forall x_{1}, x_{2} \cdot \operatorname{in}\left(n_{1}, m\left(x_{1}, x_{2}\right)\right) \equiv\left(n_{1}=x_{1}\right) \\
& \forall x_{1}, x_{2} . \operatorname{in}\left(n_{2}, m\left(x_{1}, x_{2}\right)\right) \equiv\left(n_{2}=x_{2}\right) .
\end{aligned}
$$

While this validates $\mathrm{in}\left(n_{1}, m_{1,2}\right)$, it falsifies $\mathrm{in}\left(n_{1}, m_{2}\right)$. See (22) for the general case.

We need to be able to refer to separate instances of the same default appearing in different sets. For this we introduce a binary, infix function-symbol ".". For $\delta_{j} \in D_{i}$ we write $n_{\delta_{j}} \cdot m_{i}$ or $n_{j} \cdot m_{i}$ to name the instance of $\delta_{j}$ appearing in $D_{i}$. This results in name set $N \cdot M=\{n \cdot m \mid n \in N, m \in M\}$. Corresponding axioms, as $\mathrm{DCA}_{N \cdot M}$ and $\mathrm{UNA}_{N \cdot M}$, are obtained in a straightforward way. In what follows, we refer to the various domain closure and unique names axioms pertaining to $N, M$, and $N \cdot M$ as $A x(N){ }^{9}$

Given language $\mathcal{L}$, we define a family of languages $\mathcal{L}(m)$ for $m \in M$ as follows. If $P$ is an $i$-ary predicate symbol then $P(\cdot)$ is a distinct $(i+1)$-ary predicate symbol. If $\gamma \in \mathcal{L}$ then $\gamma(m) \in \mathcal{L}(m)$ is the formula obtained by replacing all predicate symbols in $\gamma$ with predicate symbols extended as described, and with term $m$ as the $(i+1)^{s t}$ argument. This extra argument is used to index formulas by the (names of) sets in which they are used.

Lastly, we introduce special-purpose predicates for controlling the application of sets of defaults. ok, bl, and ko are the same as in Section 4. We introduce $\prec \subseteq M \times M$ as the object language counterpart of the meta-level symbol $<$. The predicate $a p(\cdot)$ is used to indicate that a rule instance or a set of rules has been applied. These are summarised in the following table:

\begin{tabular}{l|l} 
Name & Use/meaning \\
\hline$m \prec m^{\prime}$ & $D_{m}<D_{m^{\prime}}$ \\
ok $(e)$ & It is ok to try to apply set/rule $e$. \\
ap $(e)$ & Set/rule $e$ is applied. \\
bl $(m)$ & Not all rules in set $m$ can be applied. \\
ko $(m)$ & For set $m$, bl $(m)$ is true.
\end{tabular}

\footnotetext{
${ }^{8} \mathrm{We}$ could restrict the set of names to just the sets appearing in $\left.2^{D}\right|_{<}$. The present scheme however is more general and anticipates the application developed in Section 6.

${ }^{9}$ Note that names in $M$ and $N \cdot M$ are obtained from those in $N$.
} 
Lastly, for simplicity, if $\left.D \notin 2^{D}\right|_{<}$we assume the existence of a trivial, maximally preferred default $\delta_{\uparrow}=\frac{\mathrm{ok}\left(n_{\uparrow} \cdot m_{D}\right):}{\mathrm{ap}\left(n_{\uparrow} \cdot m_{D}\right)}$. As well we assume the existence of a minimally-preferred default $\delta_{\downarrow}=$ $\frac{\mathrm{ok}\left(n_{\downarrow} \cdot m_{\emptyset}\right):}{\mathrm{ap}\left(n_{\downarrow} \cdot m_{\emptyset}\right)}$. In these cases we have:

$$
D_{i}<\left\{\delta_{\uparrow}\right\} \quad \text { for every } D_{i} \neq\left\{\delta_{\uparrow}\right\}
$$

and

$$
\left\{\delta_{\downarrow}\right\}<D_{i} \quad \text { for every } D_{i} \neq\left\{\delta_{\downarrow}\right\} .
$$

This gives us (if needed) a trivial maximally preferred default that is always applicable, and a trivial minimally preferred default that can be used to "collect" results.

We let $\mathcal{L}^{+}$be the language obtained by unioning all languages $\mathcal{L}(m)$ for $m \in M$ and using the aforementioned names and introduced predicates and functions. For a set of formulas $S \subseteq \mathcal{L}^{+}$, we let $\downarrow(S)$ be the projection of formulas indexed by $m_{\emptyset}$ onto the language $\mathcal{L}$.

Definition 5.2 For $S \subseteq \mathcal{L}^{+}$, let $\downarrow(S)=\left\{\gamma \in \mathcal{L} \mid \gamma\left(m_{\emptyset}\right) \in S\right\}$.

Taking all this into account, we obtain the following translation, mapping default theories in language $\mathcal{L}$ onto default theories in the language $\mathcal{L}^{+}$.

Definition 5.3 Given a set-ordered default theory $(D, W,<)$ over $\mathcal{L}$ and its set of associated default names $N \cup \dot{\cup}$, define $\mathcal{S}((D, W,<))=\left(D^{\prime}, W^{\prime}\right)$ over $\mathcal{L}^{+}$by

$$
\begin{aligned}
D^{\prime} & =D_{N} \cup D_{M} \cup D_{\neg} \\
W^{\prime} & =W_{W} \cup W_{D} \cup W_{M} \cup W_{\prec} \cup A x(N)
\end{aligned}
$$

where

$$
\begin{aligned}
& D_{N}=\left\{\frac{\alpha(x) \wedge i n(n, x) \wedge \mathrm{ok}(n \cdot x): \beta(x)}{\gamma(x) \wedge \operatorname{ap}(n \cdot x)} \mid n: \frac{\alpha: \beta}{\gamma} \in D\right\} \\
& D_{M}=\left\{\frac{\mathrm{ok}(x): \neg \mathrm{ko}(x)}{\forall y \in N \cdot \operatorname{in}(y, x) \supset \mathrm{ok}(y \cdot x)}\right\} \\
& \cup\left\{\frac{\operatorname{in}(n, x) \wedge \mathrm{ok}(x): \neg \alpha(x)}{\mathrm{bl}(x)} \mid n: \frac{\alpha: \beta}{\gamma} \in D\right\} \\
& \cup\left\{\frac{([\forall y \in N \cdot \operatorname{in}(y, x) \supset c(y, x)] \supset \neg \beta(x)) \wedge \mathrm{ok}(x):}{\mathrm{bl}(x)} \mid n: \frac{\alpha: \beta}{\gamma} \in D\right\} \\
& D_{\neg}=\left\{\frac{: \neg(x \prec y)}{\neg(x \prec y)}, \frac{: \neg i n(x, y)}{\neg i n(x, y)}\right\} \\
& W_{W}=\{\forall x \in M . \alpha(x) \mid \alpha \in W\} \\
& W_{D}=\left\{\forall x \in M . c\left(n_{\delta}, x\right) \equiv \operatorname{CON}(\delta)(x) \mid \delta \in D\right\} \\
& W_{M}=\left\{\forall x_{1}, \ldots, x_{k} \cdot \operatorname{in}\left(n_{i}, m\left(x_{1}, \ldots, x_{k}\right)\right) \equiv\left(n_{i}=x_{i}\right) \mid n_{i} \text { in }\left\langle n_{1}, \ldots, n_{k}\right\rangle\right\} \\
& W_{\prec}=\left\{\operatorname{ok}\left(m_{D}\right)\right\} \\
& \cup \quad\left\{m_{i} \prec m_{j} \mid\left(D_{i}, D_{j}\right) \in<\right\} \\
& \cup\{\forall x \in M .[\forall y \in M .(x \prec y) \supset(\operatorname{ko}(y) \vee \operatorname{ap}(y))] \supset \text { ok }(x)\} \\
& \cup\{\forall x \in M \cdot \operatorname{bl}(x) \supset \operatorname{ko}(x)\} \\
& \cup\{\forall x \in M \cdot[\forall y \in N \cdot \operatorname{in}(y, x) \supset \text { ap }(y \cdot x)] \supset \text { ap }(x)\} \\
& \cup\left\{\forall x, x^{\prime} \in M \cdot \operatorname{ap}(x) \supset\left(\neg\left(x \prec x^{\prime}\right) \supset\left(\forall y . c(y, x) \supset c\left(y, x^{\prime}\right)\right)\right\}\right.
\end{aligned}
$$


Definition 5.4 Let $(D, W,<)$ be a set-ordered default theory over $\mathcal{L}$ and let $E$ be an extension of $\mathcal{S}((D, W,<))$. Then $\gamma$ is a set-ordered default conclusion of $(D, W,<)$ with respect to $E$ iff $\gamma \in \downarrow(E)$.

The rules in $D_{N}$ and $D_{M}$ directly generalise those in (4-6), from treating a single set named $m$ to an arbitrary set referenced by variable $x$. The specific consequents used in the second rule in (6) are dealt with via the axioms in $\left(W_{D} / 21\right)$ that allow us to quantify over default consequents (via predicate $c$ ). This trick avoids the blowup that would occur in (18) if we were to explicitly give the consequents of the rules. In Section 6 this would amount to an exponential blowup.

The rules in $\left(D_{\neg} / 19\right)$ provide us with complete knowledge on predicates $\prec$ and $i n$. The axioms in $\left(W_{W} / 20\right)$ propagate the information in $W$ to all possible contexts.

$W_{M}$ takes care of what we need with respect to set operations; that is, (22) formalises set membership. $W_{\prec}$ axiomatises the control flow along the partial order induced by $\prec$. Axiom (24) translates the (external) order $<$ on sets into a predicate $\prec$ in the language. Axioms (23) and (25) tell us when it is ok to consider a certain set: we always consider the maximum set $D$; otherwise, via (25), we consider a set just when every <-greater-set is known to be blocked or has been applied. (26) tells us when the consideration of a set is cancelled; this happens because a set is inapplicable (given by bl). (27) asserts that a set is applied just if all of its member rules are. Once we have found an applicable set of rules the consequences are propagated to all sets not explicitly $<$-greater than that set; (28) propagates the consequences.

\section{Example 1:}

Consider again our initial car example:

$$
\begin{aligned}
& n_{1}: \frac{: P}{P}, \quad n_{2}: \frac{: S}{S}, \quad n_{3}: \frac{: E}{E} . \\
& m_{\emptyset}:\left\{\delta_{\downarrow}\right\}<m_{1}:\left\{\frac{: P}{P}\right\}<m_{2}:\left\{\frac{: S}{S}\right\}<m_{3}:\left\{\frac{: E}{E}\right\}<m_{1,2}:\left\{\frac{: P}{P}, \frac{: S}{S}\right\}<m_{D}:\left\{\delta_{\uparrow}\right\}
\end{aligned}
$$

Let $W=\{\neg(P \wedge E \wedge S)\}$. We obtain a single extension containing $P\left(m_{\emptyset}\right)$ and $S\left(m_{\emptyset}\right)$ as well as $\neg E\left(m_{\emptyset}\right)$. Informally the extension is constructed as follows. ${ }^{10}$

- We have initially ok $\left(m_{D}\right)$ (23) and then also ap $\left(m_{D}\right)$, from which we derive ok $\left(m_{1,2}\right)$ (25). Thus in this last case, it is ok to consider the application of the $<$-greatest nontrivial set, (named) $m_{1,2}$.

- From this we obtain ok $\left(n_{1} \cdot m_{1,2}\right)$ and ok $\left(n_{2} \cdot m_{1,2}\right)(16)$.

That is, it is ok to consider the application of the individual default instances in set $m_{1,2}$; these are the defaults with names $n_{1} \cdot m_{1,2}$ and $n_{2} \cdot m_{1,2}$.

- If both $\delta_{1}$ and $\delta_{2}$ are applicable (which they are), then we conclude $P\left(m_{1,2}\right) \wedge \mathrm{ap}\left(n_{1} \cdot m_{1,2}\right)$ and $S\left(m_{1,2}\right) \wedge \mathrm{ap}\left(n_{1} \cdot m_{1,2}\right)(15)$ as well as ap $\left(m_{1,2}\right)(27)$.

So we obtain the conclusions from the defaults, together with an assertion that the defaults have been applied (via ap). As well, we assert that the set has been applied via ap $\left(m_{1,2}\right)$.

\footnotetext{
${ }^{10}$ More formally, the extension is verified with respect to the grounded enumeration, according to Definition 2.1.
} 
- From this we get (among other things) $P\left(m_{\emptyset}\right)$ and $S\left(m_{\emptyset}\right)$, and $P\left(m_{3}\right)$ and $S\left(m_{3}\right)$ (28). We also get ok $\left(m_{3}\right)(25)$.

So the results are propagated to (among other sets) the minimum set $m_{\emptyset}$ and the next $<-$ greatest set $m_{3}$.

- Hence (since we derive $\neg E\left(m_{3}\right)$ ) also bl $\left(m_{3}\right)$ (18) and $\mathrm{ko}\left(m_{3}\right)$ (26).

Thus we fail to be able to apply $m_{3}$.

We thus fail to derive ok $\left(n_{3} \cdot m_{3}\right)$ since the instance of (14) doesn't apply. To see that there are no other extensions, it suffices to observe that there is no way of deriving $\mathrm{bl}\left(m_{1,2}\right)$.

Second, consider where $W=\{\neg(P \wedge S)\}$, and so we cannot satisfy our topmost (nontrivial) preference. From ok $\left(m_{D}\right)$ we derive ok $\left(m_{1,2}\right)$ In view of $\neg\left(P\left(m_{1,2}\right) \wedge S\left(m_{1,2}\right)\right)$, we cannot have an extension in which both $\delta_{1}$ and $\delta_{2}$ apply, which is mirrored by the fact that we obtain bl $\left(m_{1,2}\right)$ and so ko $\left(m_{1,2}\right)$. We fail to derive ok $\left(n_{1} \cdot m_{1,2}\right)$ and ok $\left(n_{2} \cdot m_{1,2}\right)$. We subsequently obtain ok $\left(m_{3}\right)$ from which we (eventually) get $E\left(m_{3}\right)$, and $E\left(m_{\emptyset}\right)$ and ap $\left(m_{3}\right)$. From this we get ok $\left(m_{2}\right)$, (eventually) $S\left(m_{2}\right)$ and $S\left(m_{\emptyset}\right)$ and ap $\left(m_{2}\right)$. Finally we get ok $\left(m_{1}\right)$, and eventually bl $\left(m_{1}\right)$ and ko $\left(m_{1}\right)$. We obtain a single extension, containing $S\left(m_{\emptyset}\right), E\left(m_{\emptyset}\right)$, as desired.

\section{Example 2:}

The next example deals with the situation where defaults inside a set depend upon each other. We are given

$$
\begin{aligned}
& n_{1}: \frac{: S}{S}, \quad n_{2}: \frac{: T}{T}, \quad n_{3}: \frac{: Q}{Q}, \quad n_{4}: \frac{Q: R}{R} \\
& m_{\emptyset}:\left\{\delta_{\downarrow}\right\}<m_{1}:\left\{\frac{: S}{S}\right\}<m_{2}:\left\{\frac{: T}{T}\right\}<m_{3,4}:\left\{\frac{: Q}{Q}, \frac{Q: R}{R}\right\}<m_{D}:\left\{\delta_{\uparrow}\right\}
\end{aligned}
$$

Assume that we have an assertion to the effect that no more than two of $Q, R, S, T$ can hold. Having derived ap $\left(m_{D}\right)$, we obtain from $W_{\prec}$ that $\mathrm{ok}\left(m_{3,4}\right)$. We then obtain ok $\left(n_{3} \cdot m_{3,4}\right)$, and ok $\left(n_{4} \cdot m_{3,4}\right)$, which allow us to apply default $\delta_{3}$, yielding in turn $Q\left(m_{3,4}\right) \wedge$ ap $\left(n_{3} \cdot m_{3,4}\right)$. With $Q\left(m_{3,4}\right)$ at hand, we can now apply default $\delta_{4}$, yielding $R\left(m_{3,4}\right) \wedge \mathrm{ap}\left(n_{4} \cdot m_{3,4}\right)$. This allows us to deduce ap $\left(m_{3,4}\right)$. Thus, we obtain an extension containing $Q\left(m_{3,4}\right)$ and $R\left(m_{3,4}\right)$, and so $Q\left(m_{\emptyset}\right)$ and $R\left(m_{\emptyset}\right)$.

It is instructive to see how an extension with $S\left(m_{\emptyset}\right)$ and $T\left(m_{\emptyset}\right)$ is denied. Assume there is an extension $E^{\prime}$ containing $S\left(m_{\emptyset}\right)$ and $T\left(m_{\emptyset}\right)$. Then, $E^{\prime}$ contains also $\neg Q\left(m_{\emptyset}\right)$ and $\neg R\left(m_{\emptyset}\right)$, since it is deductively closed. However, we have ok $\left(m_{3,4}\right)$ and both ok $\left(n_{3} \cdot m_{3,4}\right)$ and ok $\left(n_{4} \cdot m_{3,4}\right)$ in $E^{\prime}$. Since there is no way to derive $\neg Q\left(m_{3,4}\right)$, we have that $Q\left(m_{3,4}\right)$ is consistent with $E^{\prime}$ and so the rule $\frac{\mathrm{ok}\left(n_{3} \cdot m_{3,4}\right) \wedge i n\left(n_{3}, m_{3,4}\right): Q\left(m_{3,4}\right)}{Q\left(m_{3,4}\right) \wedge \mathrm{ap}\left(n_{3} \cdot m_{3,4}\right)}$ in the image of the translation (15) is applicable. Similarly, the rule $\frac{Q\left(m_{3,4}\right) \wedge \mathrm{ok}\left(n_{4} \cdot m_{3,4}\right) \wedge i n\left(n_{4}, m_{3,4}\right): R\left(m_{3,4}\right)}{R\left(m_{3,4}\right) \wedge \operatorname{ap}\left(n_{4} \cdot m_{3,4}\right)}$ is applicable, and we obtain ap $\left(m_{3,4}\right)$ and so $Q\left(m_{\emptyset}\right)$ and $R\left(m_{\emptyset}\right)$, destroying the purported extension. Hence, $E^{\prime}$ is not an extension of the set-ordered theory. 


\section{Example 3:}

$$
m_{1}:\left\{\frac{: C}{C}\right\}<m_{2}:\left\{\frac{: B \wedge \neg C}{B}\right\}
$$

We get an extension containing $B\left(m_{\emptyset}\right)$ and $C\left(m_{\emptyset}\right)$. This is due to the fact that we have a highly prescriptive approach, in that we attempt to apply the highest ranked set(s) independently of lower-ranked defaults. Given the result of applying (or not) the highest ranked set, we attempt to apply the next highest ranked set(s), and so on. Similarly, for the following theory:

$$
m_{1}:\left\{\frac{: B}{B}\right\}<m_{2}:\left\{\frac{B: C}{C}\right\}
$$

we obtain an extension containing $B\left(m_{\emptyset}\right)$ but not $C\left(m_{\emptyset}\right)$.

Theorem 4.2 showed that any single set of defaults, treated as a set, has an extension. However, with multiple sets, as in the original approach, we are not guaranteed the existence of an extension. For example the following theory, where there are no preferences except involving $m_{D}$ and $m_{\emptyset}$, lacks an extension:

\section{Example 4:}

$$
m_{1}:\left\{\frac{: A \wedge \neg B}{A}\right\}, \quad m_{2}:\left\{\frac{: B \wedge \neg C}{B}\right\}, \quad m_{3}:\left\{\frac{: C \wedge \neg A}{C}\right\} .
$$

The following theorem summarises the major properties of our approach, and demonstrates that rules are applied in the desired order. Define $\bar{S}=\{\neg \alpha \mid \alpha \in S\}$.

Theorem 5.1 Let $E$ be a consistent extension of $\mathcal{S}((D, W,<))$ for set-ordered default theory $(D, W,<)$. We have for all $\delta \in D$ and for all $D_{m},\left.D_{m^{\prime}} \in 2^{D}\right|_{<}$that:

1. $\mathrm{ok}(m) \in E$

2. $\operatorname{ap}(m) \in E$ iff $\mathrm{ko}(m) \notin E$

3. $\mathrm{ko}(m) \in E$ iff $\mathrm{bl}(m) \in E$

4. ok $(m) \in E_{i}$ and $\operatorname{PRE}\left(D_{m}\right) \subseteq E_{j}$ and $\overline{\operatorname{JUS}\left(D_{m}\right)} \cap E=\emptyset$ implies $\operatorname{CON}\left(D_{m}\right) \subset E_{\max (i+2, j)+3}$ and $\operatorname{ap}(m) \in E_{\max (i+2, j)+3}$

5. ok $(m) \in E_{i}$ and $\operatorname{PRE}\left(D_{m}\right) \nsubseteq E$ implies $\mathrm{ko}(m) \in E_{i+2}$

6. ok $(m) \in E_{i}$ and $\overline{\operatorname{JUS}\left(D_{m}\right)} \cap E \neq \emptyset$ implies $\mathrm{ko}(m) \in E_{j}$ for some $j>i+2$

If preferences are expressed among sets with a single element only then the extensions obtained by the approach of [DSO0a] are also obtained here. For this, let $\mathcal{T}$ be the translation of ordered default theories into standard default logic given in Section 4 in [DS00a].

Theorem 5.2 Let $(D, W,<)$ be an ordered semi-normal default theory over $\mathcal{L}$ and let $\left(D, W,<^{\prime}\right)$ be the set-ordered default theory, where $<^{\prime}=\left\{\left(\{\delta\},\left\{\delta^{\prime}\right\}\right) \mid\left(\delta, \delta^{\prime}\right) \in<\right\}$.

If $E$ is an extension of $\mathcal{T}((D, W,<))$ there is an extension $E^{\prime}$ of $\mathcal{S}\left(\left(D, W,<^{\prime}\right)\right)$ such that $E \cap \mathcal{L}=\downarrow\left(E^{\prime}\right)$. 
Note that the restriction to semi-normal default theories does not limit the scope of the theorems, since any default theory is expressible as a semi-normal default theory yielding the same extension [Jan99] (cf. Section 2).

For semi-normal default theories, the approach can be made equivalent (modulo the original language) to standard default logic if there are no preferences:

Theorem 5.3 Let $(D, W)$ be a semi-normal default theory over $\mathcal{L}$. Define $<$ by: for every $\delta \in D$ we have $\left\{\delta_{\downarrow}\right\}<\{\delta\}<\left\{\delta_{\uparrow}\right\}$.

If $E$ is an extension of $(D, W)$ then there is some extension $E^{\prime}$ of $\mathcal{S}((D, W,<))$ such that $E=\downarrow\left(E^{\prime}\right)$.

If $E^{\prime}$ is an extension of $\mathcal{S}((D, W,<))$ then $E=\downarrow\left(E^{\prime}\right)$ is an extension of $(D, W)$.

The reason why our approach has a tight relationship with its predecessors in the case of seminormal default theories dealing only with singleton sets is that this case annuls the contextual information in the rule

$$
\frac{([\forall y \in N \cdot \operatorname{in}(y, x) \supset c(y, x)] \supset \neg \beta(x)) \wedge \mathrm{ok}(x):}{\operatorname{bl}(x)}
$$

That is, given a singleton set containing a semi-normal default rule, viz.

$$
m:\left\{\frac{\alpha: \beta \wedge \gamma}{\gamma}\right\}
$$

the translated rule is equivalent to

$$
\frac{[\gamma(m) \supset \neg(\beta(m) \wedge \gamma(m))] \wedge \text { ok }(m):}{\mathrm{bl}(m)} \quad \text { which reduces to } \frac{\neg(\beta(m) \wedge \gamma(m)) \wedge \text { ok }(m):}{\mathrm{bl}(m)},
$$

which is the rule obtained in our original approach, except for the fact that it uses a set name instead of a rule name.

\section{Encoding extensions using sets}

In this section we use the machinery developed in the previous sections to encode the extensions of a semi-monotonic default theory $(D, W) \cdot{ }^{11}$ We modify the definitions of the last section so that, viewed algorithmically, the translated theory carries out the following: If the original set of defaults $D$ constitutes the set of generating defaults of an extension, then a corresponding "ap"literal is derived, all default consequences are obtained, and all subsets of the set of defaults are rendered inapplicable. If this isn't the case (and $D$ isn't a set of generating defaults), we proceed along the partial order induced by set inclusion and consider every set $D \backslash\{\delta\}$ for every $\delta \in D$ to see whether it is a set of generating defaults. Any time a set of generating defaults is found, all subsets of that set are rendered inapplicable.

Our naming conventions are unchanged; in particular we use the same "vector-oriented" representation for naming sets and for capturing set membership. As before, given language $\mathcal{L}$, we

\footnotetext{
${ }^{11}$ This section is a précis of results in [DS02].
} 
define a family of languages $\mathcal{L}(m)$ for $m \in M$, and the language $\mathcal{L}^{+}$by unioning all languages $\mathcal{L}(m)$ for $m \in M$.

Our central definition is much the same as Definition 5.3. Differences are noted by underlining the appropriate part of an equation.

Definition 6.1 Given a finite default theory $(D, W)$ over $\mathcal{L}$ and its set of associated default names $N$, define $\mathcal{E}((D, W))=\left(D^{\prime}, W^{\prime}\right)$ over $\mathcal{L}^{+}$by

$$
\begin{aligned}
D^{\prime} & =D_{N} \cup D_{M} \cup D_{\neg} \\
W^{\prime} & =W_{W} \cup W_{D} \cup W_{M} \cup W_{\prec} \cup A x(N)
\end{aligned}
$$

where

$$
\begin{aligned}
& D_{N}=\left\{\frac{\alpha(x) \wedge i n(n, x) \wedge \mathrm{ok}(n \cdot x): \beta(x)}{\gamma(x) \wedge \operatorname{ap}(n \cdot x)} \mid n: \frac{\alpha: \beta}{\gamma} \in D\right\} \\
& D_{M}=\left\{\frac{\mathrm{ok}(x): \neg \mathrm{ko}(x)}{\forall y \in N \cdot \operatorname{in}(y, x) \supset \mathrm{ok}(y \cdot x)}\right\} \\
& \cup\left\{\frac{\operatorname{in}(n, x) \wedge \mathrm{ok}(x): \neg \alpha(x)}{\mathrm{bl}(x)} \mid n: \frac{\alpha: \beta}{\gamma} \in D\right\} \\
& \cup\left\{\frac{([\forall y \in N \cdot \operatorname{in}(y, x) \supset c(y, x)] \supset \neg \beta(x)) \wedge \mathrm{ok}(x):}{\mathrm{bl}(x)} \mid n: \frac{\alpha: \beta}{\gamma} \in D\right\} \\
& D_{\neg}=\left\{\frac{: \neg(x \prec y)}{\neg(x \prec y)}, \frac{: \neg i n(x, y)}{\neg i n(x, y)}\right\} \\
& W_{W}=\{\forall x \in M . \alpha(x) \mid \alpha \in W\} \\
& W_{D}=\left\{\forall x \in M . c\left(n_{\delta}, x\right) \equiv \operatorname{CON}(\delta)(x) \mid \delta \in D\right\} \\
& W_{M}=\left\{\forall x_{1}, \ldots, x_{k} . \operatorname{in}\left(n_{i}, m\left(x_{1}, \ldots, x_{k}\right)\right) \equiv\left(n_{i}=x_{i}\right) \mid n_{i} \text { in }\left\langle n_{1}, \ldots, n_{k}\right\rangle\right\} \\
& \cup\left\{\underline{\forall x, x^{\prime} \in M .\left(\left[\exists y \in N . \neg i n(y, x) \wedge i n\left(y, x^{\prime}\right)\right]\right.}\right. \\
& \left.\left.\wedge\left[\forall y \cdot \operatorname{in}(y, x) \supset \operatorname{in}\left(y, x^{\prime}\right)\right]\right) \supset x \prec x^{\prime}\right\} \\
& W_{\prec}=\left\{\operatorname{ok}\left(m_{D}\right)\right\} \\
& \cup\{\forall x \in M .(\forall y \in M . x \prec y \supset \underline{\mathrm{bl}(y)}) \supset \mathrm{ok}(x)\} \\
& \cup \quad\{\forall x \in M \cdot \operatorname{bl}(x) \supset \operatorname{ko}(x)\} \\
& \cup\{\forall x \in M .(\forall y \in N . \operatorname{in}(y, x) \supset \text { ap }(y \cdot x)) \supset \operatorname{ap}(x)\} \\
& \cup\left\{\forall x, x^{\prime} \in M . \underline{\operatorname{ap}(x) \supset\left(x^{\prime} \prec x \supset \operatorname{ko}\left(x^{\prime}\right)\right)}\right\}
\end{aligned}
$$

$W_{M}$ is augmented so that as well as formalising set membership, (41) formalises strict set inclusion. $W_{\prec}$ axiomatises the control flow along the partial order induced by $\prec$, but now implicitly specified via set containment. For (43), we consider a set to see whether it is applicable just when every superset is determined to be inapplicable. (44) tells us one case when we don't consider a set: when it is found to be blocked. Once we have found an applicable set of rules (and hence a set of generating defaults) we need not consider any subset; (46) cancels the consideration of all such subsets and so constitutes a second case in which a set is not considered.

For example, consider the following normal default theory:

$$
\Delta=\left(\left\{n_{1}: \frac{: A}{A}, n_{2}: \frac{: B}{B}, n_{3}: \frac{: \neg B}{\neg B}, n_{4}: \frac{B: D}{D}\right\}, \emptyset\right) .
$$

From our translated theory, we get an extension where the only "ap-literals" applied to set names are ap $\left(m_{1,2,4}\right)$ and ap $\left(m_{1,3}\right)$. That is, $\Delta$ has two extensions, the first with generating defaults $\delta_{1}$, 
$\delta_{2}, \delta_{4}$, and the second with $\delta_{1}, \delta_{3}$. Among formulas in the extension of the translated theory are $A\left(m_{1,2,4}\right), A\left(m_{1,3}\right), B\left(m_{1,2,4}\right), \neg B\left(m_{1,3}\right)$, and $D\left(m_{1,2,4}\right)$.

The next results show that our default theories resulting from $\mathcal{E}$ have appropriate properties.

Theorem 6.1 Let $E$ be a consistent extension of $\mathcal{E}((D, W))$ for semi-monotonic default theory $(D, W)$. We have for all $\delta \in D$ and for all $D_{m}, D_{m^{\prime}} \subseteq D$ that:

1. ok $(m) \in E$ iff $(\operatorname{ap}(m) \in E$ or bl $(m) \in E)$

2. $\operatorname{ap}(m) \in E$ iff $\mathrm{ko}(m) \notin E$

3. If $\mathrm{ap}(m) \in E$ then $\mathrm{bl}\left(m^{\prime}\right) \in E$ for all $m^{\prime} \in M$ with $m \prec m^{\prime} \in E$

4. If $\mathrm{ap}(m) \in$ E then $\mathrm{ko}\left(m^{\prime}\right) \in$ E for all $m^{\prime} \in M$ with $m^{\prime} \prec m \in E$

5. If $\operatorname{ap}(m), \operatorname{ap}\left(m^{\prime}\right) \in E$ then $\neg\left(m \prec m^{\prime}\right) \in E$

6. ok $(m) \in E_{i}$ and $\operatorname{PRE}\left(D_{m}\right) \cup \operatorname{CON}\left(D_{m}\right) \subseteq E_{j}$ and $\overline{\operatorname{JUS}\left(D_{m}\right)} \cap E=\emptyset$ implies ap $(m) \in$ $E_{\max (i+2, j)+3}$

7. ok $(m) \in E_{i}$ and $\operatorname{PRE}\left(D_{m}\right) \nsubseteq E$ implies bl $(m) \in E_{i+1}$

8. ok $(m) \in E_{i}$ and $\overline{\operatorname{JUS}\left(D_{m}\right)} \cap E \neq \emptyset$ implies $\mathrm{bl}(m) \in E_{j}$ for some $j>i+1$

Thus, for example, in parts 3 and 4 of the theorem, if a set of defaults with name $m$ is applied (and so is a set of generating defaults), then every superset has been determined to be blocked, and every subset is explicitly eliminated. The only sets explicitly considered (part 1) are those determined to be blocked or applied.

The next two theorems show that our translation captures an encoding of extensions of a semimonotonic default theory.

Theorem 6.2 Let $(D, W)$ be a semi-monotonic default theory with extensions $E_{1}, \ldots, E_{n}$ and let $E$ be an extension of $\mathcal{E}((D, W))$.

Then, for any $i \in\{1, \ldots, n\}$, there is some $m \in M$ naming $G D\left(D, E_{i}\right)$ such that $\operatorname{ap}(m) \in E$.

As a consequence we obtain:

Corollary 6.1 If $(D, W)$ is a semi-monotonic default theory then $\mathcal{E}((D, W))$ has a unique extension.

The next theorem provides a converse to the preceding.

Theorem 6.3 Let $(D, W)$ be a semi-monotonic default theory and let $E$ be the extension of $\mathcal{E}((D, W))$.

Then for any $\operatorname{ap}(m) \in E$ with $m \in M$, we have that $T h(\{\gamma \mid \gamma(m) \in E\})$ is an extension of $(D, W)$. 
Lastly, our claim that a translated theory is "almost" a constant factor larger than the original requires elaboration. $\mathrm{UNA}_{N}$ yields a quadratic number of unique names assertions. In practice this is no problem, since any sensible implementation would not explicitly list such axioms. With the exception of unique names assertions, a translated theory is a constant factor larger than the original. To see this, it suffices to examine Definition 6.1 . Each of $(33,35,36,39,40)$ introduce $|D|$ axioms/rules; (38) introduces $|W|$ axioms. All remaining terms introduce a single axiom. Moreover, the size of individual axioms is similarly bounded. (For example, each instance of (33) is a constant factor larger than the original default.)

\subsection{Discussion}

The fact that we can encode all extensions of a semi-monotonic theory within a single extension means that we can now encode phenomena of interest, usually dealt with at the metalevel, at the object level. Specifically we can now encode the notions of skeptical and credulous inference within a theory. In order to do this, we introduce two new constants skep and cred, for "skeptical" and "credulous" respectively.

A formula is a skeptical inference if it is a member of every extension. In our approach, this means that it follows in every "ap-set". Hence we define skeptical inference within a theory, for a given formula $\gamma$, by

$$
(\forall x \in M \cdot \operatorname{ap}(x) \supset \gamma(x)) \supset \gamma(\text { skep }) .
$$

For credulous inference there are a number of possibilities. The simplest is to assert that a formula is a credulous inference if it is a member of some extension:

$$
(\exists x \in M . \operatorname{ap}(x) \wedge \gamma(x)) \supset \gamma(\text { cred }) .
$$

With this definition, a formula and its negation may be credulous inferences; hence it is not a very useful definition, since it can lead to an inconsistent extension. While there are ways to get around this (for instance by making cred a predicate applied to quoted formulas) one can also employ a stronger definition. One stronger definition is to assert that a formula is a credulous inference if it is a member of some extension, and its negation is a member of no extension. We can define this notion of credulous inference (indicated by $\mathrm{cred}^{\prime}$ ) for a formula $\gamma$ by means of the default:

$$
\frac{\exists x \in M \cdot \operatorname{ap}(x) \wedge \gamma(x): \forall x \in M \cdot \operatorname{ap}(x) \supset \gamma(x)}{\gamma\left(\text { cred }^{\prime}\right)} .
$$

Hence in Example (47), we obtain that $A$ is a skep inference, while $D$ is a $c r e d^{\prime}$ inference. $B$ and $\neg B$ are cred inferences. There are other possibilities for defining credulous inference. The point here isn't to evaluate possibilities per se; rather it is to suggest that such possibilities can be encoded within a theory.

Although our results are restricted to semi-monotonic theories, such theories nonetheless can be used to capture an interesting set of problems. We have already suggested that the approach may be applicable in diagnosis programs, such as found in [Rei87]. Similarly, the approach can be used to directly encode applications expressible in Theorist [Poo88]. That is, there is a correspondence between so-called Poole-type theories and Theorist with constraints [Dix92]. Since Poole-type 
theories are semi-monotonic, this means that our approach can encode any application encodable in Theorist.

Our approach relies on a first-order language. Despite this, the image of a theory over a finite language remains finite. Concerning a possible implementation, however, it is not advisable to use a bottom-up grounding approach, as done in many implementations of extended logic programming [ELM ${ }^{+}$97, NS97]. Instead, a query-oriented approach seems to be preferable, since it may rely on unification rather than ground instantiation.

\section{Conclusion}

We have presented a general approach for representing and reasoning about preferences among sets of defaults in default logic. To begin with, we first considered how to control the application of a set of defaults so that either all rules apply (if possible) or none do (if not). From this we showed how we could deal with preferences among sets of defaults, and subsequently how the approach could be used to encode extensions of semi-monotonic default theories in the object language.

The main approach begins with an ordered theory, consisting of a set of world knowledge, a set of defaults, and a set of preference relations among subsets of the defaults. This theory is translated into a second, standard default theory wherein the preferences are respected: sets of defaults are "applied" according to the given order; for a set of order-equivalent defaults, either all are applied, or none are. Using constants and functions for naming, we can refer to default rules, sets of defaults, and instances of a rule in a set. Via these names we can, first, determine whether a set of defaults is its own set of generating defaults and, second, consider the application of sets of defaults ordered by set containment. The translated theory requires a modest increase in space: except for unique names axioms, only a constant-factor increase is needed. The translated theory is a (regular, Reiter) default theory. Hence we essentially axiomatise the notion of "extensions" for a class of default theories in a single extension. Further, we are able to prove that our translation behaves correctly.

Although a specific approach is described for reasoning with preferences over sets, we don't suggest that this is the only approach. Rather we are also interested in also proposing a general methodology for dealing with (here) preferences among sets of defaults, but that is also more generally applicable [DS00a, DS00b]. That is, one has some informal intuitions concerning some phenomenon of interest. Given this, these intuitions are formalised by providing a translation of one theory (encoding said phenomenon) into a second, such that properties of the target phenomenon provably hold. Clearly, one could use this methodology to develop other mechanisms for dealing, for example, with preferences over sets.

To the best of our knowledge, our approach is the first approach to deal with preferences among sets of defaults. Unlike most previous approaches to dealing with preferences, we do not generate extensions and select those with the most preferred applied rules; rather extensions containing the most preferred applied rules are directly generated. Another major point of contrast with previous work concerning preferences is that we remain within the framework of standard default logic (rather than building a scheme on top of default logic). This has several advantages. First, our approach can be immediately implemented by making use of an existing default logic theorem prover such as DeRes [CMT96]. Second, in "compiling" preferences into default logic, and in 
using the standard machinery of default logic, we obtain insight into the notion of preference orderings. In a sense the translation provides an "axiomatisation" of the sense of preference that we wish to capture. Last, it is easier to compare differing approaches to handling such orderings, since we remain within the same "base" framework. Thus, by examining the respective definitions, it is clear how the main definitions of Sections 5 and 6 differ. As a point of theoretical interest, we show that incorporating explicit prescriptive priorities among sets of rules in default logic provides no real increase in the expressibility of default logic. Using the approach for encoding extensions, we can now express notions such as skeptical and credulous inference within a theory. Arguably this will prove beneficial in expressing at the object level problems and approaches generally expressed at the metalevel. Areas of application range from specific areas such as diagnosis, to broadlyapplicable approaches such as Theorist.

\section{Acknowledgements}

THe first author was supported by a Canadian NSERC Discovery Grant. The second author was supported by the German Science Foundation (DFG) under grant SCHA 550/6, TP C.

\section{A Proofs for Section 4}

The following definition is used in the proofs.

Definition A.1 ([Rei80]) Let $(D, W)$ be a default theory. For any set of formulas $S$, let $\Gamma(S)$ be the smallest set of formulas $S^{\prime}$ such that

1. $W \subseteq S^{\prime}$,

2. $\operatorname{Th}\left(S^{\prime}\right)=S^{\prime}$,

3. For any $\frac{\alpha: \beta}{\gamma} \in D$, if $\alpha \in S^{\prime}$ and $\neg \beta \notin S$ then $\gamma \in S^{\prime}$.

A set of formulas $E$ is an extension of $(D, W)$ if $\Gamma(E)=E$.

With respect to the translations in Definitions 4.1, 5.3, and 6.1, we adopt the following notation: For a set of defaults with name $m$ and one of its members with name $n$, let $\delta_{a}^{m}, \delta_{b_{1}}^{m, n}$, and $\delta_{b_{2}}^{m, n}$ be the corresponding default rules in $D_{M}$. Let $\delta_{a}^{m, n}$ denote the transform of the individual default named $n$ with $x$ instantiated to $m$ in $D_{N}$.

\section{Proof 4.1}

1. if part Suppose $\operatorname{ap}\left(n_{\delta}\right) \in E$ for all $\delta \in D_{m}$. Since $E$ is deductively closed and since $E$ contains Formula (10), we deduce that $\operatorname{ap}(m) \in E$.

only-if part Suppose $\operatorname{ap}(m) \in E$. By construction, this implies ap $\left(n_{\delta}\right) \in E$ for all $\delta$ such that $i n\left(n_{\delta}, m\right) \in E$, or $\delta \in D_{m}$. By the definition of $D_{N}$ and $W_{D}$, however, we have $\operatorname{ap}\left(n_{\delta}\right) \in E$ only if $\operatorname{CON}(\delta) \wedge \operatorname{ap}\left(n_{\delta}\right) \in E$. Since this holds for all $\delta \in D_{m}$, we obtain $\left\{\operatorname{ap}\left(n_{\delta}\right) \mid \delta \in D_{m}\right\} \cup \operatorname{CON}\left(D_{m}\right) \subseteq E$. 
2. if part Suppose $\left\{\operatorname{ap}\left(n_{\delta}\right) \mid \delta \in D_{m}\right\} \nsubseteq E$. Thus, there is some $\delta \in D_{m}$ such that $\delta_{a}^{m, n_{\delta}} \notin$ $G D\left(D^{\prime}, E\right)$. Then, one of the following cases must be true.

- If $\neg J U S(\delta) \in E$, then clearly from $W_{D}$ we get $\operatorname{CON}\left(\delta_{1}\right) \wedge \ldots \wedge \operatorname{CON}\left(\delta_{k}\right) \supset$ $\neg J U S(\delta) \in E$, where $\left\{\delta_{1}, \ldots, \delta_{k}\right\}=D_{m}$. By Theorem 4.1.3 and the fact that $E$ is deductively closed, we get that

$$
\left[\operatorname{CON}\left(\delta_{1}\right) \wedge \ldots \wedge \operatorname{CON}\left(\delta_{k}\right) \supset \neg J U S(\delta)\right] \wedge \text { ok }(m) \in E .
$$

Hence $\delta_{b_{2}}^{m, n_{\delta}} \in G D\left(D^{\prime}, E\right)$, that is, bl $(m) \in E$.

- Suppose $\operatorname{PRE}(\delta) \wedge \mathrm{ok}\left(n_{\delta}\right) \notin E$. Since $E$ is deductively closed, we may distinguish the following cases.

- Assume $\operatorname{PRE}(\delta) \notin E$. Consequently, $\delta_{b_{1}}^{m, n_{\delta}} \in G D\left(D^{\prime}, E\right)$, that is, bl $(m) \in E$.

- If ok $\left(n_{\delta}\right) \notin E$, then $\delta_{a}^{m} \notin G D\left(D^{\prime}, E\right)$, since this is the only means by which we can fail to obtain ok $\left(n_{\delta}\right) \in E$. Hence $\operatorname{ko}(m) \in E$; hence from (9) we get $\mathrm{bl}(m) \in E$.

Thus, in all cases we obtain that $\mathrm{bl}(m) \in E$.

only-if part Suppose $\mathrm{bl}(m) \in E$. We distinguish the following two cases.

- If $\delta_{b_{1}}^{m, n_{j}} \in G D\left(D^{\prime}, E\right)$, then we have that $\operatorname{PRE}\left(\delta_{j}\right) \notin E$ for some $\delta_{j} \in D_{m}$. Therefore, $\left(\delta_{j}\right)_{a}^{m, n_{j}} \notin G D\left(D^{\prime}, E\right)$ and clearly ap $\left(n_{j}\right) \notin E$.

- If $\delta_{b_{2}}^{m, n_{j}} \in G D\left(D^{\prime}, E\right)$, then we have for some $\delta_{j} \in\left\{\delta_{1}, \ldots, \delta_{k}\right\}=D_{m}$ that

$$
\operatorname{CON}\left(\delta_{1}\right) \wedge \ldots \wedge \operatorname{CON}\left(\delta_{k}\right) \supset \neg J U S\left(\delta_{j}\right) \in E .
$$

Assume $\left\{\operatorname{ap}\left(n_{\delta}\right) \mid \delta \in D_{m}\right\} \subseteq E$, that is, by definition of $D_{N}$ that $\{\operatorname{CON}(\delta) \wedge$ $\left.\operatorname{ap}\left(n_{\delta}\right) \mid \delta \in D_{m}\right\} \subseteq E$. Since $E$ is deductively closed we get from (48) that $\neg J U S\left(\delta_{j}\right) \in E$ and therefore $\left(\delta_{j}\right)_{a}^{m, n_{j}} \notin G D\left(D^{\prime}, E\right)$ and clearly ap $\left(n_{j}\right) \notin E$, a contradiction.

In both cases we thus obtain $\left\{\operatorname{ap}\left(n_{\delta}\right) \mid \delta \in D_{m}\right\} \nsubseteq E$.

3. if part Suppose $\operatorname{ap}\left(n_{\delta}\right) \in E$. Then we have necessarily that $\delta_{a}^{m, n_{\delta}} \in G D\left(D^{\prime}, E\right)$, and therefore that $\operatorname{PRE}(\delta) \wedge \mathrm{ok}\left(n_{\delta}\right) \in E$, and so ok $\left(n_{\delta}\right) \in E$.

only-if part Suppose ok $\left(n_{\delta}\right) \in E$. Then, we have by definition of $D_{M}$ that

$$
\frac{: \neg \mathrm{ko}(m)}{\mathrm{ok}\left(n_{1}\right) \wedge \ldots \wedge \mathrm{ok}\left(n_{\delta}\right) \wedge \ldots \wedge \mathrm{ok}\left(n_{k}\right)} \in G D\left(D^{\prime}, E\right) .
$$

Clearly, we thus have $\mathrm{ko}(m) \notin E$; this implies bl $(m) \notin E$. As a consequence, we get $\delta_{b_{1}}^{m, n_{\delta}} \notin G D\left(D^{\prime}, E\right)$ and $\delta_{b_{2}}^{m, n_{\delta}} \notin G D\left(D^{\prime}, E\right)$. We obtain for each $\delta \in\left\{\delta_{1}, \ldots, \delta_{k}\right\}=$ $D_{m}$ that

$$
\operatorname{PRE}(\delta) \in E \quad \text { and } \quad \operatorname{CON}\left(\delta_{1}\right) \wedge \ldots \wedge \operatorname{CON}\left(\delta_{k}\right) \supset \neg J U S(\delta) \notin E .
$$

Furthermore, the latter gives $\neg J U S(\delta) \notin E$. With ok $\left(n_{1}\right) \wedge \ldots \wedge$ ok $\left(n_{\delta}\right) \wedge \ldots \wedge$ ok $\left(n_{k}\right) \in$ $E$ and the fact that $E$ is deductively closed, we get that $\delta_{a}^{m, n_{\delta}} \in G D\left(D^{\prime}, E\right)$ for all $\delta \in D_{m}$. That is, since $E$ is deductively closed, ap $\left(n_{\delta}\right) \in E$ for all $\delta \in D_{m}$.

4. if part Suppose $\mathrm{ko}(m) \notin E$, and so $\mathrm{bl}(m) \notin E$. As a consequence, we get $\delta_{b_{1}}^{m, n_{\delta}} \notin$ $G D\left(D^{\prime}, E\right)$ and $\delta_{b_{2}}^{m, n_{\delta}} \notin G D\left(D^{\prime}, E\right)$ for some $\delta \in D_{m}$. As a corollary of Theorem 4.1.1-2, we obtain that: $\delta_{a}^{m} \in G D\left(D^{\prime}, E\right)$ iff $\left(\delta_{b_{1}}^{m, n_{\delta}} \notin G D\left(D^{\prime}, E\right)\right.$ and $\delta_{b_{2}}^{m, n_{\delta}} \notin$ 
$G D\left(D^{\prime}, E\right)$ for all $\left.\delta \in D_{m}\right)$. This implies that $\delta_{a}^{m} \in G D\left(D^{\prime}, E\right)$. Therefore ok $\left(n_{\delta}\right) \in$ $E$.

only-if part If ok $\left(n_{\delta}\right) \in E$, then $\delta_{a}^{m} \in G D\left(D^{\prime}, E\right)$, that is, $\mathrm{ko}(m) \notin E$.

5. The if-part is trivial.

For the only-if part, assume that ap $\left(n_{\delta}\right) \in E$ for some $\delta \in D_{m}$. Then $\delta_{a}^{m, n_{\delta}} \in G D\left(D^{\prime}, E\right)$ and therefore $\delta_{a}^{m} \in G D\left(D^{\prime}, E\right)$.

We also have $i n\left(n_{\delta}, m\right) \in E$ for all $\delta \in D_{m}$. Further, $\delta_{a}^{m} \in G D\left(D^{\prime}, E\right) \operatorname{implies}$ ok $\left(n_{\delta}\right) \in E$ for all $\delta \in D_{m}$. By Theorem 4.1.3, this implies ap $\left(n_{\delta}\right) \in E$ for all $\delta \in D_{m}$.

\section{Proof 4.2}

1. First, assume that default theory $(\emptyset \cup D, W)$ has an extension $E$ where $G D(D, E)=D$.

From Definition 2.1 we have that $E=\bigcup_{i=0}^{\infty} E_{i}$ where

$$
\begin{aligned}
E_{0} & =W \\
E_{i+1} & =\operatorname{Th}\left(E_{i}\right) \cup\left\{\gamma \mid \frac{\alpha: \beta}{\gamma} \in D, \alpha \in E_{i}, \neg \beta \notin E\right\} .
\end{aligned}
$$

Obviously then $E=\bigcup_{i=0}^{\infty} E_{i}$ where

$$
\begin{aligned}
E_{0} & =W \cup\left\{\operatorname{ok}\left(n_{1}\right) \wedge \ldots \wedge \text { ok }\left(n_{k}\right)\right\} \\
E_{i+1} & =\operatorname{Th}\left(E_{i}\right) \cup\left\{\gamma \mid \frac{\alpha: \beta}{\gamma} \in D, \alpha \in E_{i}, \neg \beta \notin E\right\} .
\end{aligned}
$$

defines an extension of $\left(D_{N}, W \cup\left\{\mathrm{ok}\left(n_{1}\right) \wedge \ldots \wedge \mathrm{ok}\left(n_{k}\right)\right\}\right)$.

Replacing $W$ with $W^{\prime}$ in the above defines an extension of $\left(D_{N}, W^{\prime} \cup\left\{\operatorname{ok}\left(n_{1}\right) \wedge \ldots \wedge\right.\right.$ ok $\left.\left.\left(n_{k}\right)\right\}\right)$ as well as of $\left(D_{N} \cup D_{M}, W^{\prime} \cup\left\{\operatorname{ok}\left(n_{1}\right) \wedge \ldots \wedge \operatorname{ok}\left(n_{k}\right)\right\}\right)$ or $\left(D^{\prime}, W^{\prime} \cup\left\{\operatorname{ok}\left(n_{1}\right) \wedge \ldots \wedge \operatorname{ok}\left(n_{k}\right)\right\}\right)$

From this it follows that

$$
\begin{aligned}
E_{-1}^{\prime} & =W^{\prime} \\
E_{0}^{\prime} & =\operatorname{Th}\left(W^{\prime}\right) \cup\left\{\operatorname{ok}\left(n_{1}\right) \wedge \ldots \wedge \text { ok }\left(n_{k}\right)\right\} \\
& =\operatorname{Th}\left(E_{-1}\right) \cup\left\{\gamma \mid \frac{\alpha: \beta}{\gamma} \in D^{\prime}, \alpha \in E_{-1}, \neg \beta \notin E^{\prime}\right\} \\
E_{i+1}^{\prime} & =\operatorname{Th}\left(E_{i}^{\prime}\right) \cup\left\{\gamma \mid \frac{\alpha: \beta}{\gamma} \in D^{\prime}, \alpha \in E_{i}^{\prime}, \neg \beta \notin E^{\prime}\right\} \quad \text { for } i>1 .
\end{aligned}
$$

and $E^{\prime}=\bigcup_{i=-1}^{\infty} E_{i}^{\prime}$ defines an extension of $\left(D^{\prime}, W^{\prime}\right)$.

Thus for this case we have that $\mathcal{S}_{m}((\emptyset \cup D, W))$ has extension $E$ where $E \cap \mathcal{L}=$ $\operatorname{Th}(W \cup \operatorname{CON}(D))$.

(Note for this case that having $\mathrm{bl}(m)$ in our purported extension $E^{\prime}$ would contradict the assumption that $D$ is a set of generating defaults for $(\emptyset \cup D, W)$. 
2. Assume that $D$ is not a set of generating defaults for default theory $(\emptyset \cup D, W)$.

Thus for any set $E$ and for

$$
\begin{aligned}
E_{0} & =W \\
E_{i+1} & =\operatorname{Th}\left(E_{i}\right) \cup\left\{\gamma \mid \frac{\alpha: \beta}{\gamma} \in D, \alpha \in E_{i}, \neg \beta \notin E\right\} .
\end{aligned}
$$

we have that $E \neq \bigcup_{i=0}^{\infty} E_{i}$.

In particular this holds for $E=T h(W \cup \operatorname{CON}(D))$.

Since $\bigcup_{i=0}^{\infty} E_{i}=T h(W \cup C)$ for some $C \subset C O N(D)$, this means that some default $\delta_{j} \in D$ fails to apply. There are two possibilities:

(a) $\alpha_{j} \notin E_{i}$ for every $i \geq 0$.

(b) $\neg \beta_{j} \in E$.

For the first case, assume that there is an extension $E^{\prime}$ of $\mathcal{S}_{m}((\emptyset \cup D, W))$ containing $\alpha_{j}$. Since $\alpha_{j} \notin W^{\prime} \backslash W$ we have that $W \cup C^{\prime} \vdash \alpha_{j}$ for some $C^{\prime} \subset \operatorname{CON}(D)$.

Since $W \vdash \alpha_{j}$ contradicts $\alpha_{j} \notin E_{i}$ above, we have that $C^{\prime} \neq \emptyset$ and hence ap $(n) \in E^{\prime}$ for some default $n: \delta$.

From Theorem 4.1.5 we obtain that $\left\{\operatorname{ap}\left(n_{\delta}\right) \mid \delta \in D\right\} \subseteq E^{\prime}$, hence in particular ap $\left(n_{j}\right) \in E^{\prime}$ and so $\alpha_{j} \in G D_{i}$, a contradiction.

Hence there is no extension $E^{\prime}$ of $\mathcal{S}_{m}((\emptyset \cup D, W))$ containing $\alpha_{j}$.

It follows that $\operatorname{Th}\left(\left\{W^{\prime}, \mathrm{bl}(m), \mathrm{ok}\left(n_{1}\right) \wedge \ldots \wedge\right.\right.$ ok $\left.\left.\left(n_{k}\right)\right\}\right)$ is an extension of $\mathcal{S}_{m}((\emptyset \cup D, W))$ : we have shown that $\alpha_{j} \in E^{\prime}$ is not possible for any purported extension. Hence $\delta_{b_{1}}^{m, n_{j}}$ does apply, yielding $\mathrm{bl}(m)$, and $\mathrm{ko}(m)$. This then prevents $\delta_{a}^{m}$ and any of $\delta_{a}^{m, n}$ from applying.

In the second case, where $\neg \beta_{j} \in E$, since $E=\operatorname{Th}(W \cup \operatorname{CON}(D))$ we get that $W \cup$ $\operatorname{CON}(D) \vdash \neg \beta_{j}$ which, by the previous argument, gives an extension $\mathcal{S}_{m}((\emptyset \cup D, W))$ by virtue of the applicability of $\delta_{b_{2}}^{m, n_{j}}$

\section{Proof 4.3}

This follows immediately from the first part of the proof of Theorem 4.2.

\section{B Proofs for Section 5}

We first show the following results:

Lemma 1 Let $E$ be a consistent extension of $\mathcal{S}((D, W,<))=\left(D^{\prime}, W^{\prime}\right)$ for set-ordered default theory $(D, W,<)$.

1. $\left(m \prec m^{\prime}\right) \in E$ iff $\neg\left(m \prec m^{\prime}\right) \notin E$ 
2. in $\left(n_{\delta}, m\right) \in E$ iff $\neg$ in $\left(n_{\delta}, m\right) \notin E$

\section{Proof 1}

1. By the consistency of $E$, we cannot have both $m \prec m^{\prime} \in E$ and $\neg\left(m \prec m^{\prime}\right) \in E$.

Assume that for some $D_{m}, D_{m^{\prime}} \subseteq D$, we have neither $m \prec m^{\prime} \in E$ nor $\neg\left(m \prec m^{\prime}\right) \in E$.

Then, however, the default rule $\frac{: \neg\left(m \prec m^{\prime}\right)}{\neg\left(m \prec m^{\prime}\right)}$ in $D_{\neg}$ is applicable and we obtain $\neg\left(m \prec m^{\prime}\right) \in E$, which contradicts our assumption.

We have thus shown that $m \prec m^{\prime} \in E$ iff $\neg\left(m \prec m^{\prime}\right) \notin E$.

2. Analogous to Proof 1.1 .

For further proofs, we observe moreover the following complementary propositions.

Lemma 2 Let $E$ be a consistent extension of $\mathcal{S}((D, W,<))=\left(D^{\prime}, W^{\prime}\right)$ for set-ordered default theory $(D, W,<)$.

1. We have for all $D_{1}, D_{2} \subseteq D$ that $\left(m_{1} \prec m_{2}\right) \in E$ iff $\left(m_{1} \prec m_{2}\right) \in W^{\prime}$.

2. We have for all $D_{m} \subseteq D$ and $\delta \in D$ that in $\left(n_{\delta}, m\right) \in E$ iff $i n\left(n_{\delta}, m\right) \in W^{\prime}$.

\section{Proof 2}

1. Clearly, we have $\left(m_{1} \prec m_{2}\right) \in E$ if $\left(m_{1} \prec m_{2}\right) \in W^{\prime}$.

Assume we have $\left(m_{1} \prec m_{2}\right) \in E$ and $\left(m_{1} \prec m_{2}\right) \notin W^{\prime}$. Since $\left(m_{1} \prec m_{2}\right) \notin W^{\prime}=E_{0}$, there must exist (according to Definition 2.1) some $i \geq 0$ with $\left(m_{1} \prec m_{2}\right) \notin E_{i}$ but $\left(m_{1} \prec m_{2}\right) \in E_{i+1}$. Since there are no default rules with consequents containing positive occurrences of $\prec$-literals, we must have $\left(m_{1} \prec m_{2}\right) \in T h\left(E_{i}\right)$. For the same reason, all positive occurrences in $E_{i}$ must stem from $W_{\prec}$. In fact, all positive occurrences of $\prec$-literals in $W_{\prec}$ (in clause form) come from (24) or (25) in $W_{\prec}$. For (24), we obtain $\left(m_{1} \prec m_{2}\right) \in W^{\prime}$, a contradiction. (25) can be written in the form $\left(\left(m_{1} \prec m_{2}\right) \wedge \phi\right) \vee \varphi \vee$ ok $\left(m_{1}\right)$ for some formulas $\phi, \varphi$. A proof for $E_{i} \vdash\left(m_{1} \prec m_{2}\right)$ must thus contain the negative ok-literal ok $\left(m_{1}\right)$. There are however no negative occurrences of ok-literals in $\mathcal{S}((D, W,<))$, neither in $D^{\prime}$ nor in $W^{\prime}$, a contraction.

2. Analogous to proof of Lemma 2.1.

\section{Proof 5.1}

3. For (3) we observe that if $\mathrm{bl}(m) \in E$ then $\mathrm{ko}(m) \in E$ via (26). Since there are no positive occurrences of ko in $\mathcal{S}((D, W,<))$ we conclude bl $(m) \in E$ iff $\operatorname{ko}(m) \in E$.

1+2. We show for all $\left.D_{m} \in 2^{D}\right|_{<}$by induction on $<$that ok $(m) \in E$ and either ap $(m) \in E$ or $\mathrm{bl}(m) \in E$ 
Base By definition, ok $\left(m_{D}\right) \in W_{\prec} \subseteq E$. Also, $\perp \notin E$, since $E$ is consistent. Therefore, we have $\frac{(T \supset \perp) \wedge \text { ok }\left(m_{D}\right):}{\mathrm{bl}\left(m_{D}\right)} \notin G D\left(D^{\prime}, E\right)$ and $\frac{\mathrm{ok}\left(m_{D}\right): \perp}{\mathrm{bl}\left(m_{D}\right)} \notin G D\left(D^{\prime}, E\right)$. That is, $\mathrm{bl}\left(m_{D}\right) \notin E$. As a consequence, we have $\mathrm{ko}\left(m_{D}\right) \notin E$, as shown above.

Since ok $\left(m_{D}\right) \in E$ and $\mathrm{ko}\left(m_{D}\right) \notin E$, we have $\frac{\mathrm{ok}\left(m_{D}\right): \neg \mathrm{ko}\left(m_{D}\right)}{\mathrm{ok}\left(n_{\uparrow} \cdot m_{D}\right)} \in G D\left(D^{\prime}, E\right)$. Clearly, then we also have $\frac{T \wedge i n\left(n_{\uparrow}, m_{D}\right) \wedge \text { ok }\left(n_{\uparrow} \cdot m_{D}\right): T}{T \wedge a p\left(n_{\uparrow} \cdot m_{D}\right)} \in G D\left(D^{\prime}, E\right)$.

By definition, $i n\left(n_{\uparrow}, m_{D}\right) \in E$. By Lemma $2, \neg i n\left(n_{\delta}, m_{D}\right) \in E$ for all $\delta \in D \backslash\left\{\delta_{\top}\right\}$. Since $E$ is deductively closed and since $E$ contains the formula (27), we deduce that $\mathrm{ap}\left(m_{D}\right) \in E$.

Step Consider $\left.D_{m} \in 2^{D}\right|_{<}$and assume that for all $D_{m^{\prime}}$ with $D_{m}<D_{m^{\prime}}$ we have ok $\left(m^{\prime}\right) \in$ $E$ and ap $\left(m^{\prime}\right) \in E$ iff $\mathrm{ko}\left(m^{\prime}\right) \notin E$ - that is iff $\mathrm{bl}\left(m^{\prime}\right) \notin E$.

First, we have the following lemma.

Lemma 3 Given the induction hypothesis, we have ok $(m) \in E$.

Proof 3 By the induction hypothesis, we have ap $\left(m^{\prime}\right) \in E$ iff bl $\left(m^{\prime}\right) \notin E$ for all $D_{m^{\prime}}$ with $D_{m}<D_{m^{\prime}}$.

By definition of $W_{\prec}$ and Lemma 1 , we have $m \prec m^{\prime} \in E$ for all $D_{m}, D_{m^{\prime}}$ with $D_{m}<D_{m^{\prime}}$.

Analogously, we get $\left(m \prec m^{\prime}\right) \notin E$ for all $D_{m}, D_{m^{\prime}}$ with $D_{m} \nless D_{m^{\prime}}$. From this, we get by means of $D_{\neg}$ that $\neg\left(m \prec m^{\prime}\right) \in E$ for all $D_{m}, D_{m^{\prime}}$ with $D_{m} \nless D_{m^{\prime}}$.

Because $E$ is deductively closed and contains (25), and because bl $(y) \in E$ iff $\operatorname{ko}(y) \in E$, we deduce that $\mathrm{ok}(m) \in E$.

For $D_{m}=\left.\left\{\delta_{j} \mid j=1 . . k\right\} \in 2^{D}\right|_{<}$, we distinguish the following cases.

- If $\frac{\mathrm{ok}(m):-\mathrm{ko}(m)}{\mathrm{ok}\left(n_{1} \cdot m\right) \wedge \wedge . . \wedge \mathrm{ok}\left(n_{k} \cdot m\right)} \in G D\left(D^{\prime}, E\right)$, then ok $(m) \in E$ and $\mathrm{ko}(m) \notin E$. The latter implies bl $(m) \notin E$. As a consequence, we get $\delta_{b_{1}}^{m, n_{j}} \notin G D\left(D^{\prime}, E\right)$ and $\delta_{b_{2}}^{m, n_{j}} \notin$ $G D\left(D^{\prime}, E\right)$ for $j=1 . . k$. Since ok $\left(n_{1} \cdot m\right) \wedge \ldots \wedge$ ok $\left(n_{k} \cdot m\right) \in E$, we thus have for each $\delta_{j} \in\left\{\delta_{1}, \ldots, \delta_{k}\right\}=D_{m}$ that

$$
\text { and } \quad \begin{array}{ll}
\operatorname{PON}\left(\delta_{j}\right) \in E \\
\operatorname{CON}\left(\delta_{1}\right) \wedge \ldots \wedge \operatorname{CON}\left(\delta_{k}\right) \supset \neg \operatorname{JUS}\left(\delta_{j}\right) \notin E .
\end{array}
$$

Furthermore, (49) implies $\neg J U S\left(\delta_{j}\right) \notin E$. With ok $\left(n_{1} \cdot m\right) \wedge \ldots \wedge$ ok $\left(n_{k} \cdot m\right) \in E$ and the fact that $E$ is deductively closed, we get that $\left(\delta_{j}\right)_{a}^{m, n} \in G D\left(D^{\prime}, E\right)$ for $j=1 . . k$. That is, since $E$ is deductively closed, $\operatorname{ap}\left(n_{j} \cdot m\right) \in E$ for $j=1 . . k$. And since $E$ is deductively closed and since $E$ contains the formula (27), we deduce that $\mathrm{ap}\left(m_{D}\right) \in E$.

- If $\frac{\mathrm{ok}(m):-\mathrm{ko}(m)}{\mathrm{ok}\left(n_{1} \cdot m\right) \wedge \ldots \wedge \mathrm{ok}\left(n_{k} \cdot m\right)} \notin G D\left(D^{\prime}, E\right)$, then $\mathrm{ko}(m) \in E$, since ok $(m) \in E$ by Lemma 3. The former implies that $\mathrm{bl}(m) \in E$.

Finally we have $\operatorname{ap}(m) \notin E$ : If $\mathrm{ap}(m) \in E$, this can only be derived via Formula (27). But this means that $\mathrm{ap}\left(n_{j} \cdot m\right) \in E$ for every $\delta_{j} \in D_{m}$; hence ok $\left(n_{j} \cdot m\right) \in E$ for every $\delta_{j} \in D_{m}$. But ok $\left(n_{j} \cdot m\right)$ is obtainable only from application of the default $\frac{\mathrm{ok}\left(m_{i}\right):-\mathrm{ko}\left(m_{i}\right)}{\mathrm{ok}\left(n_{1} \cdot m_{i}\right) \wedge \ldots \wedge \mathrm{ok}\left(n_{k} \cdot m_{i}\right)}$, contradiction.

This demonstrates that ap $(m) \in E$ iff bl $(m) \in E$ for all $\left.D_{m} \in 2^{D}\right|_{<}$. 
4. Suppose ok $(m) \in E_{i}$ and $\operatorname{PRE}\left(D_{m}\right) \cup \operatorname{CON}\left(D_{m}\right) \subseteq E_{j}$ and $\overline{J U S\left(D_{m}\right)} \cap E=\emptyset$.

According to the two last statements, we have for all $\delta \in D_{m}$ that $\operatorname{PRE}(\delta) \in E_{j}$ and $\neg J U S(\delta) \notin E$. While the former implies immediately that $\delta_{b_{1}}^{m, n_{\delta}} \notin G D\left(D^{\prime}, E\right)$, the latter implies $\delta_{b_{2}}^{m, n_{\delta}} \notin G D\left(D^{\prime}, E\right)$. Hence, bl $(m) \notin E$ and furthermore $\mathrm{ko}(m) \notin E$, since $\mathrm{ko}(m)$ is only derivable from $\mathrm{bl}(m)$.

Together, ok $(m) \in E_{i}$ and $\mathrm{ko}(m) \notin E$ imply that $\frac{\mathrm{ok}(m):-\operatorname{ko}(m)}{\mathrm{ok}\left(n_{1} \cdot m\right) \wedge \ldots \wedge \mathrm{ok}\left(n_{k} \cdot m\right)} \in G D_{i}$, that is, ok $\left(n_{1} \cdot m\right) \wedge \ldots \wedge$ ok $\left(n_{k} \cdot m\right) \in E_{i+1}$, and thus ok $\left(n_{l} \cdot m\right) \in E_{i+2}$ for all $l=1 . . k$. Since $\operatorname{PRE}\left(\delta_{l}\right) \in E_{j}$, we get $\operatorname{PRE}\left(\delta_{l}\right) \wedge \mathrm{ok}\left(n_{l} \cdot m\right) \in E_{\max (i+2, j)+1}$. This implies together with $\neg J U S\left(\delta_{l}\right) \notin E$ that $\left(\delta_{l}\right)_{a}^{m, n_{l}} \in G D_{\max (i+2, j)+1}$, hence $C O N\left(\delta_{l}\right) \wedge$ ap $\left(n_{l} \cdot m\right) \in E_{\max (i+2, j)+2}$. Accordingly, $\operatorname{ap}\left(n_{l} \cdot m\right) \in E_{\max (i+2, j)+3}$ for all $l=1 . . k$.

Since $i n\left(\delta_{l}, m\right) \in E_{0}$ for all $l=1 . . k$ and $\neg i n(\delta, m) \in E_{1}$ for $\delta \in D \backslash D_{m}$ according to Lemma 2, we obtain by means of Formula (27), also in $E_{0}$, that ap $(m) \in E_{\max (i+2, j)+3}$.

5. We are given ok $(m) \in E_{i}$ and $\operatorname{PRE}\left(D_{m}\right) \nsubseteq E$, that is, $\operatorname{PRE}(\delta) \notin E$ for some $\delta \in D_{m}$. Therefore $\delta_{b_{1}}^{m, n_{\delta}} \in G D_{i}$ and therefore $\mathrm{bl}(m) \in E_{i+1}$ and $\mathrm{ko}(m) \in E_{i+2}$.

6. Suppose ok $(m) \in E_{i}$ and $\overline{J U S\left(D_{m}\right)} \cap E \neq \emptyset$, that is, $\neg J U S(\delta) \in E$ for some $\delta \in D_{m}$. Assume $\neg J U S(\delta) \in E_{k-1}$ for some minimal $k$. Consequently, we have

$$
\operatorname{CON}\left(\delta_{1}\right) \wedge \ldots \wedge \operatorname{CON}\left(\delta_{k}\right) \supset \neg J U S(\delta) \in E_{k} \quad \text { where } D_{m}=\left\{\delta_{1}, \ldots, \delta_{k}\right\} .
$$

Then, we get $\operatorname{PRE}\left(\delta_{b_{2}}^{m, n_{\delta}}\right) \in E_{\max (i, k)+1}$. This implies $\delta_{b_{2}}^{m, n_{\delta}} \in G D_{\max (i, k)+1}$, and furthermore $\mathrm{bl}(m) \in E_{\max (i, k)+2}$ and $\mathrm{ko}(m) \in E_{\max (i, k)+3}$. That is, $\operatorname{ko}(m) \in E_{j}$ for some $j>i+2$.

Proof 5.2 We start by fixing the components of the different default theories:

Define $\mathcal{T}((D, W,<))=\left(D^{t}, W^{t}\right)$,

$$
\begin{aligned}
D^{t} & =\left\{\delta_{a}, \delta_{b_{1}}, \delta_{b_{2}} \mid \delta \in D\right\} \cup D_{\prec}^{t} \\
W^{t} & =W \cup W_{\prec}^{t} \cup\left\{\mathrm{DCA}_{N}, \mathrm{UNA}_{N}\right\}
\end{aligned}
$$

where $W_{\prec}^{t}$ and $D_{\prec}^{t}$ are defined as their unindexed counterparts $W_{\prec}$ and $D_{\prec}$, respectively, in Definition 4.1 of [DS00a]. Because of the fact that $D$ is a set of semi-normal default rules and $<^{\prime}=\left\{\left(\{\delta\},\left\{\delta^{\prime}\right\}\right) \mid\left(\delta, \delta^{\prime}\right) \in<\right\}$, we have that

$$
\delta_{a}=\frac{\alpha \wedge \mathrm{ok}\left(n_{\delta}\right): \gamma \wedge \beta}{\gamma \wedge \mathrm{ap}\left(n_{\delta}\right)}, \quad \delta_{b_{1}}=\frac{\mathrm{ok}\left(n_{\delta}\right): \neg \alpha}{\mathrm{bl}\left(n_{\delta}\right)}, \quad \delta_{b_{2}}=\frac{\neg(\gamma \wedge \beta) \wedge \mathrm{ok}\left(n_{\delta}\right):}{\mathrm{bl}\left(n_{\delta}\right)} .
$$

Similarly, define $\mathcal{S}\left(\left(D, W,<^{\prime}\right)\right)=\left(D^{s}, W^{s}\right)$,

$$
\begin{aligned}
D^{s} & =\left\{\delta_{a}^{m, n_{\delta}} \mid \delta \in D\right\} \cup\left\{\delta_{a}^{m}, \delta_{b_{1}}^{m, n_{\delta}}, \delta_{b_{2}}^{m, n_{\delta}}\left|\{\delta\}=D_{m} \in 2^{D}\right|_{<}\right\} \cup D_{\neg}^{s} \\
W^{s} & =W_{D}^{s} \cup W_{W}^{s} \cup W_{M}^{s} \cup W_{\prec}^{s} \cup A x^{s}(N)
\end{aligned}
$$

where $W_{D}^{s}, W_{W}^{s}, W_{M}^{s}, W_{\prec}^{s}, A x^{s}(N)$, and $D_{\neg}^{s}$ are defined as their unindexed counterparts $W_{D}$, $W_{W}, W_{M}, W_{\prec}, A x(N)$, and $D_{\neg}$, respectively, in Definition 5.3. 
We simplify the rules in $D^{s}$ as follows. We omit the first superscript $m$ for $\delta_{a}^{m, n_{\delta}}, \delta_{b_{1}}^{m, n_{\delta}}$ and $\delta_{b_{2}}^{m, n_{\delta}}$ since we deal with singleton sets. We thus write $\delta_{a}^{n_{\delta}}, \delta_{b_{1}}^{n_{\delta}}$ and $\delta_{b_{2}}^{n_{\delta}}$. We take $n_{\delta}: D_{\delta}=\{\delta\}$. Similarly for naming: rather than write the name of the rule instance in $\{\delta\}$ as $n_{\delta} \cdot m_{\delta}$ we can use, with no ambiguity, $n_{\delta}$. Finally, we replace $c(m, n)$ by $C O N(n)$. For the rules in $D^{s}$ we obtain: ${ }^{12}$

$$
\begin{array}{ll}
\delta_{a}^{n_{\delta}}=\frac{\alpha\left(m_{\delta}\right) \wedge i n\left(n_{\delta}, m_{\delta}\right) \wedge \mathrm{ok}\left(n_{\delta}\right): \gamma\left(m_{\delta}\right) \wedge \beta\left(m_{\delta}\right)}{\gamma\left(m_{\delta}\right) \wedge \mathrm{ap}\left(n_{\delta}\right)}, & \delta_{a}^{m}=\frac{\mathrm{ok}\left(m_{\delta}\right): \neg \mathrm{ko}\left(m_{\delta}\right)}{\mathrm{ok}\left(n_{\delta}\right)}, \\
\delta_{b_{1}}^{n_{\delta}}=\frac{\mathrm{ok}\left(m_{\delta}\right): \neg \alpha\left(m_{\delta}\right)}{\mathrm{bl}\left(m_{\delta}\right)}, & \delta_{b_{2}}^{n_{\delta}}=\frac{\neg\left(\gamma\left(m_{\delta}\right) \wedge \beta\left(m_{\delta}\right)\right) \wedge \mathrm{ok}\left(m_{\delta}\right):}{\mathrm{bl}\left(m_{\delta}\right)} .
\end{array}
$$

Let $E$ be an extension of $\mathcal{T}((D, W,<))$. Define

$$
\begin{aligned}
E^{\prime}=T h\left(W^{s} \cup\right. & \left\{\operatorname{ok}\left(m_{\delta}\right) \mid \delta \in D\right\} \\
\cup & \left\{\operatorname{ok}\left(n_{\delta}\right) \mid \delta_{a} \in G D\left(D^{t}, E\right)\right\} \\
\cup & \left\{C O N(\delta)\left(m_{\delta}\right) \wedge \operatorname{ap}\left(n_{\delta}\right), \operatorname{ap}\left(m_{\delta}\right) \mid \delta_{a} \in G D\left(D^{t}, E\right)\right\} \\
\cup & \left\{\operatorname{bl}\left(m_{\delta}\right), \operatorname{ko}\left(m_{\delta}\right) \mid \delta_{b_{i}} \in G D\left(D^{t}, E\right), i=1,2\right\} \\
\cup & \left\{\neg i n\left(n_{\delta}, m_{\delta^{\prime}}\right) \mid D_{\delta^{\prime}} \neq\{\delta\}\right\} \\
\cup & \left.\left\{\neg\left(m_{\delta} \prec m_{\delta^{\prime}}\right) \mid \frac{: \neg\left(n_{\delta} \prec n_{\delta^{\prime}}\right)}{\neg\left(n_{\delta} \prec n_{\delta^{\prime}}\right)} \in G D(D, E)\right\}\right)
\end{aligned}
$$

First, we show that $E \cap \mathcal{L}=\downarrow\left(E^{\prime}\right)$. We distinguish three cases.

- Consider $v \in W$.

Since $W \subseteq E \cap \mathcal{L}$ and $W \subseteq \downarrow\left(E^{\prime}\right)$ (since $W^{s} \subseteq E^{\prime}$ and $\left.W_{W}^{s} \subseteq W^{s}\right)$, this implies $v \in E \cap \mathcal{L}$ iff $v \in E^{\prime} \cap \mathcal{L}$.

- Consider $v \in \operatorname{CON}\left(G D\left(D^{t}, E\right)\right)$.

We have $\operatorname{CON}\left(G D\left(D^{t}, E\right)\right) \subseteq E \cap \mathcal{L}$ and $\operatorname{CON}\left(G D\left(D^{t}, E\right)\right) \subseteq \downarrow\left(E^{\prime}\right)$; thus $v \in E \cap \mathcal{L}$ iff $v \in E^{\prime} \cap \mathcal{L}$.

- Consider $v \in \operatorname{Th}\left(W \cup \operatorname{CON}\left(G D\left(D^{t}, E\right)\right)\right)$.

We have $W \cup \operatorname{CON}\left(G D\left(D^{t}, E\right)\right) \subseteq E \cap \mathcal{L}$ and $W \cup \operatorname{CON}\left(G D\left(D^{t}, E\right)\right) \subseteq \downarrow\left(E^{\prime}\right)$. The fact that $E$ and $E^{\prime}$ are deductively closed imply that $v \in \operatorname{Th}\left(W \cup \operatorname{CON}\left(G D\left(D^{t}, E\right)\right)\right) \subseteq E \cap \mathcal{L}$ iff $v \in \operatorname{Th}\left(W \cup \operatorname{CON}\left(G D\left(D^{t}, E\right)\right)\right) \subseteq \downarrow\left(E^{\prime}\right)$.

We have thus shown that for all $v \in \mathcal{L}$ that

$$
v \in E \cap \mathcal{L} \text { iff } v \in \downarrow\left(E^{\prime}\right) .
$$

We draw on this fact in the sequel.

If $E$ is an inconsistent extension then $E^{\prime}$ is an inconsistent extension too, since such an inconsistency is rooted in $W$ [Rei80]. We thus assume that $E$ is consistent.

We show that $E^{\prime}$ is an extension of $\mathcal{S}\left(\left(D, W,<^{\prime}\right)\right)$.

For this, we first show the following three propositions:

1. $W^{s} \subseteq E^{\prime}$, by definition.

2. $\operatorname{Th}\left(E^{\prime}\right)=E^{\prime}$, by definition.

\footnotetext{
${ }^{12}$ Note that $\delta_{a}^{m}$ and $\delta_{b_{2}}^{n_{\delta}}$ are simplified, in conformity with the preceding abbreviations.
} 
3. For any $\delta \in D^{s}$, if $\operatorname{PRE}(\delta) \in E^{\prime}$ and $\neg J U S(\delta) \notin E^{\prime}$ then $\operatorname{CON}(\delta) \in E^{\prime}$.

To show this, suppose $P R E(\delta) \in E^{\prime}$ and $\neg J U S(\delta) \notin E^{\prime}$.

- If $\delta=\frac{: \neg i n(n, m)}{\neg i n(n, m)}$ then $\operatorname{CON}(\delta) \in E^{\prime}$, for $n \neq m$ by definition of $E^{\prime}$.

- If $\delta=\frac{: \neg\left(m_{\delta} \prec m_{\delta^{\prime}}\right)}{\neg\left(m_{\delta} \prec m_{\delta^{\prime}}\right)}$ then $\operatorname{CON}(\delta) \in E^{\prime}$, by definition of $E^{\prime}$.

- If $\delta=\delta_{a}^{n_{\delta}}=\frac{\alpha\left(m_{\delta}\right) \wedge i n\left(n_{\delta}, m_{\delta}\right) \wedge \mathrm{ok}\left(n_{\delta}\right): \beta\left(m_{\delta}\right)}{\gamma\left(m_{\delta}\right) \wedge \mathrm{ap}\left(n_{\delta}\right)}$, then $\alpha\left(m_{\delta}\right) \wedge i n\left(n_{\delta}, m_{\delta}\right) \wedge \mathrm{ok}\left(n_{\delta}\right) \in E^{\prime}$. and $\neg \beta\left(m_{\delta}\right) \notin E^{\prime}$.

Claim: $\neg \beta\left(m_{\emptyset}\right) \notin E^{\prime}$.

Proof of Claim: Assume to the contrary that $\neg \beta\left(m_{\emptyset}\right) \in E^{\prime}$. So $\neg \beta \in E$ using (54).

Since $E$ is an extension of $\mathcal{T}((D, W,<))$, we have that $E \cap \mathcal{L}$ is a $<$-preserving extension of $(D, W)$ (Theorem 4.4 and Definition 4.2 of [DS00a]).

Let $\delta^{-1} \in D$ have image $\delta$ in $\mathcal{S}\left(\left(D, W,<^{\prime}\right)\right)$. Then $\beta=J U S\left(\delta^{-1}\right)$.

We have (Definition 4.2 of [DS00a]) that there exists a grounded enumeration $\left\langle\delta_{i}\right\rangle_{i \in I}$ of $G D(D, E \cap \mathcal{L})$ such that for all $i, j \in I$ and $\delta^{-1} \in D \backslash G D(D, E \cap \mathcal{L})$, we have that

(a) if $\delta_{i}<\delta_{j}$ then $j<i$ and

(b) if $\delta_{i}<\delta^{-1}$ then $\operatorname{PRE}\left(\delta^{-1}\right) \notin E$ or $W \cup \operatorname{CON}\left(\left\{\delta_{0}, \ldots, \delta_{i-1}\right\}\right) \vdash \neg J U S\left(\delta^{-1}\right)$.

Clearly $\delta^{-1} \notin G D(D, E \cap \mathcal{L})$ since $\neg \beta \in E$. As well, since $\alpha\left(m_{\delta}\right) \in E^{\prime}$, so $\alpha\left(m_{\emptyset}\right) \in E^{\prime}$, thus via (54) $\alpha \in E$, i.e. $\operatorname{PRE}\left(\delta^{-1}\right) \in E$. This means that $W \cup$ $\operatorname{CON}\left(\left\{\delta_{0}, \ldots, \delta_{i-1}\right\}\right) \vdash \neg J U S\left(\delta^{-1}\right)$ where $\delta_{k} \nless \delta^{-1}$ for $0 \leq k<i$.

Now $W \cup \operatorname{CON}\left(\left\{\delta_{0}, \ldots, \delta_{i-1}\right\}\right) \subseteq E \cap \mathcal{L}$. Thus $\neg J U S\left(\delta^{-1}\right) \in E \cap \mathcal{L}$ since $E$ is deductively closed. Via (54) we get that $\neg J U S\left(\delta^{-1}\right)\left(m_{\emptyset}\right) \in \downarrow\left(E^{\prime}\right)$. But this means that $\neg J U S\left(\delta^{-1}\right)\left(m_{\emptyset}\right)=\neg J U S(\delta) \in E^{\prime}$, contradiction. This establishes that $\neg \beta\left(m_{\emptyset}\right) \notin E^{\prime}$.

With (54), we obtain $\alpha \in E$ and $\neg \beta \notin E$. Since $E$ is an extension of $\mathcal{T}((D, W,<))$, we obtain by Theorem 4.1.2 of [DS00a] that $\mathrm{ok}\left(n_{\delta}\right) \in E$. Since $E$ is deductively closed, we have moreover $\alpha \wedge$ ok $\left(n_{\delta}\right) \in E$. We thus get $\delta_{a} \in G D\left(D^{t}, E\right)$, which implies $\gamma\left(m_{\delta}\right) \wedge \operatorname{ap}\left(n_{\delta}\right) \in E^{\prime}$ by definition of $E^{\prime}$; and so $\operatorname{CON}(\delta) \in E^{\prime}$.

- If $\delta=\delta_{a}^{m}$, then ok $\left(m_{\delta}\right) \in E^{\prime}$ and $\mathrm{ko}\left(m_{\delta}\right) \notin E^{\prime}$; the latter implies $\delta_{b_{1}} \notin G D\left(D^{t}, E\right)$ and $\delta_{b_{2}} \notin G D\left(D^{t}, E\right)$. By Theorem 4.1.8 of [DS00a], this implies $\delta_{a} \in G D\left(D^{t}, E\right)$. Hence, ok $\left(n_{\delta}\right) \in E^{\prime}$ by definition of $E^{\prime}$. That is, $\operatorname{CON}(\delta) \in E^{\prime}$.

- If $\delta=\delta_{b_{1}}^{n_{\delta}}=\frac{\mathrm{ok}\left(m_{\delta}\right): \neg \alpha\left(m_{\delta}\right)}{\mathrm{bl}\left(m_{\delta}\right)}$ then ok $\left(m_{\delta}\right) \in E^{\prime}$ and $\alpha\left(m_{\delta}\right) \notin E^{\prime}$.

We have that $\alpha\left(m_{\emptyset}\right) \notin E^{\prime}$ by an argument analogous to that in the preceding Claim: If instead we had $\alpha\left(m_{\emptyset}\right) \in E^{\prime}$ then by (54) we would have $\alpha \in E$. Thus via Theorem 4.4 of [DS00a] we have $W \cup\left\{\operatorname{CON}\left(\delta^{\prime}\right) \mid \delta^{\prime} \nless \delta_{n}\right\} \vdash \alpha$. But then via the last formula in $W_{\prec}$ we obtain $W^{s} \cup\left\{\operatorname{CON}\left(\delta^{\prime}\right)\left(m_{\emptyset}^{\prime}\right) \mid \delta^{\prime} \nless \delta_{n}\right\} \vdash \alpha\left(m_{\emptyset}^{\prime}\right)$, contradiction. So $\alpha\left(m_{\emptyset}\right) \notin E^{\prime}$.

With (54), we obtain $\alpha \notin E$. Since $E$ is an extension of $\mathcal{T}((D, W,<))$, we obtain by Theorem 4.1.2 of [DS00a] that ok $\left(n_{\delta}\right) \in E$. We thus get $\delta_{b_{1}} \in G D\left(D^{t}, E\right)$, which implies $\mathrm{bl}\left(m_{\delta}\right) \in E^{\prime}$ by definition of $E^{\prime}$. That is, $\operatorname{CON}(\delta) \in E^{\prime}$. 
- If $\delta=\delta_{b_{2}}^{n_{\delta}}=\frac{\neg\left(\gamma\left(m_{\delta}\right) \wedge \beta\left(m_{\delta}\right)\right) \wedge \mathrm{ok}\left(m_{\delta}\right):}{\mathrm{bl}\left(m_{\delta}\right)}$ then $\neg\left(\gamma\left(m_{\delta}\right) \wedge \beta\left(m_{\delta}\right)\right) \wedge$ ok $\left(m_{\delta}\right) \in E^{\prime}$. Since $E^{\prime}$ is deductively closed we have $\neg\left(\gamma\left(m_{\delta}\right) \wedge \beta\left(m_{\delta}\right)\right) \in E^{\prime}$ and so $\neg\left(\gamma\left(m_{\emptyset}\right) \wedge \beta\left(m_{\emptyset}\right)\right) \in E^{\prime}$. With (54) and since we have a semi-normal theory, we obtain $\neg \beta \in E$. Since $E$ is an extension of $\mathcal{T}((D, W,<))$, we obtain by Theorem 4.1 .2 of [DS00a] that ok $\left(n_{\delta}\right) \in E$. Since $E$ is deductively closed we have moreover $\neg \beta \wedge$ ok $\left(n_{\delta}\right) \in E$. We thus get $\delta_{b_{2}} \in$ $G D\left(D^{t}, E\right)$, which implies bl $\left(m_{\delta}\right) \in E^{\prime}$ by definition of $E^{\prime}$. That is, $\operatorname{CON}(\delta) \in E^{\prime}$.

The preceding covers all defaults $\delta \in D^{s}$ and so we have shown that $\operatorname{CON}(\delta) \in E^{\prime}$.

According to Definition A.1, we get $\Gamma\left(E^{\prime}\right) \subseteq E^{\prime}$ by minimality of $\Gamma\left(E^{\prime}\right)$.

To show the reverse, assume that $E^{\prime} \nsubseteq \Gamma\left(E^{\prime}\right)$. Consider $v \in E^{\prime}$ and assume $v \notin \Gamma\left(E^{\prime}\right)$. We distinguish the following cases.

- If $v \in W^{s}$, then $v \in \Gamma\left(E^{\prime}\right)$, since $W^{s} \subseteq \Gamma\left(E^{\prime}\right)$, a contradiction.

- If $v \in\left\{\neg\left(m_{\delta} \prec m_{\delta^{\prime}}\right) \mid \frac{: \neg\left(n_{\delta} \prec n_{\delta^{\prime}}\right)}{\neg\left(n_{\delta} \prec n_{\delta^{\prime}}\right)} \in G D\left(D^{t}, E\right)\right\}$, then we have $\left(n_{\delta} \prec n_{\delta^{\prime}}\right) \notin E$. Accordingly, $\left(m_{\delta} \prec m_{\delta^{\prime}}\right) \notin E^{\prime}$ by definition of $<^{\prime}$ and $E^{\prime}$. Hence, we infer from $\frac{: \neg\left(m_{\delta} \prec m_{\delta^{\prime}}\right)}{\neg\left(m_{\delta} \prec m_{\delta^{\prime}}\right)} \in D^{s}$ that $\neg\left(m_{\delta} \prec m_{\delta^{\prime}}\right) \in \Gamma\left(E^{\prime}\right)$, a contradiction.

- If $v \in\left\{\neg i n\left(n_{\delta}, m_{1}\right) \mid D_{1} \neq\{\delta\}\right\}$, then we have $i n\left(n_{\delta}, m_{1}\right) \notin E^{\prime}$ by the construction of $E^{\prime}$. Hence, we infer from $\frac{: \neg i n\left(n_{\delta}, m_{1}\right)}{\neg i n\left(n_{\delta}, m_{1}\right)} \in D^{s}$ that $\neg i n\left(n_{\delta}, m_{1}\right) \in \Gamma\left(E^{\prime}\right)$, a contradiction.

- We proceed by induction on the grounded enumeration $\left\langle\delta_{i}\right\rangle_{i \in I}$ of $G D\left(D^{t}, E\right)$ for

$$
\begin{aligned}
& v \in \quad\left\{\operatorname{bl}\left(m_{\delta}\right), \operatorname{ko}\left(m_{\delta}\right) \mid \delta_{b_{i}} \in G D\left(D^{t}, E\right), i=1,2\right\} \\
& \cup\left\{\operatorname{ok}\left(n_{\delta}\right), \operatorname{CON}(\delta) \wedge \operatorname{ap}\left(n_{\delta}\right), \operatorname{ap}\left(m_{\delta}\right) \mid \delta_{a} \in G D\left(D^{t}, E\right)\right\}
\end{aligned}
$$

We show that for all $\delta_{i}, i \in I$ such that $\delta_{i}=(\delta)_{a}$ or $\delta_{i}=(\delta)_{b_{j}}$ for some $j=1,2$ and some $\delta \in$ $D$ implies that either $\left\{\operatorname{ok}\left(n_{\delta}\right), C O N(\delta) \wedge \operatorname{ap}\left(n_{\delta}\right), \operatorname{ap}\left(m_{\delta}\right)\right\} \subseteq \Gamma\left(E^{\prime}\right)$ or $\left\{\operatorname{bl}\left(m_{\delta}\right), \operatorname{ko}\left(m_{\delta}\right)\right\} \subseteq$ $\Gamma\left(E^{\prime}\right)$ respectively.

First, we have the following lemma.

Lemma 4 Given the induction hypothesis, we have ok $\left(m_{\delta}\right) \in \Gamma\left(E^{\prime}\right)$.

Proof 4 Analogous to Proof of Lemma A.2 in [DS00a].

Base Clearly, $\delta_{0}=\left(\delta_{\uparrow}\right)_{a} \cdot{ }^{13}$

By definition, ok $\left(m_{D}\right) \in \Gamma\left(E^{\prime}\right)$; also $\mathrm{ko}\left(m_{D}\right) \notin E^{\prime}$ by definition of $E^{\prime}$, which together implies that ok $\left(n_{\uparrow}\right) \in \Gamma\left(E^{\prime}\right)$, which implies that $C O N\left(\delta_{\uparrow}\right) \wedge$ ap $\left(n_{\uparrow}\right) \in \Gamma\left(E^{\prime}\right)$.

Further, we have $\operatorname{in}\left(n_{\uparrow}, m_{D}\right) \in W^{s} \subseteq \Gamma\left(E^{\prime}\right)$, and by construction of $E^{\prime}$ that $i n\left(n_{\uparrow}, m_{k}\right) \notin E^{\prime}$ for $k \neq \uparrow$. Hence, we infer from $\frac{: \neg i n\left(n_{\uparrow}, m_{k}\right)}{\neg i n\left(n_{\uparrow}, m_{k}\right)} \in D^{s}$ that $\neg i n\left(n_{\uparrow}, m_{k}\right) \in$ $\Gamma\left(E^{\prime}\right)$ for $k \neq \uparrow$.

Since $\Gamma\left(E^{\prime}\right)$ is deductively closed and since $\Gamma\left(E^{\prime}\right)$ contains Formula (27), we deduce that $\operatorname{ap}\left(m_{D}\right) \in \Gamma\left(E^{\prime}\right)$.

\footnotetext{
${ }^{13} \delta_{\uparrow}$ was called $\delta_{\top}$ in [DSO0a].
} 
Step Consider $\delta_{i}$ and assume the appropriate induction hypothesis for all $\delta_{j}$ with $j<i$.

Since $\left\langle\delta_{i}\right\rangle_{i \in I}$ is grounded in $W^{t}$, we have $W^{t} \cup \operatorname{CON}\left(\left\{\delta_{0}, \ldots, \delta_{i-1}\right\}\right) \vdash \operatorname{PRE}\left(\delta_{i}\right)$. Let $\operatorname{PRE}\left(\delta_{i}\right)=\phi \wedge$ ok $(n)$ for some $\phi \in \mathcal{L}$ and some $n \in N$ and where we have name $m_{\delta}:\left\{\delta_{n}\right\}$. Then, we clearly have $W \cup \operatorname{CON}\left(\left\{\delta_{0}, \ldots, \delta_{i-1}\right\}\right) \vdash \phi$ and by monotonicity $W^{s} \cup \operatorname{CON}\left(\left\{\delta\left(m_{\delta}\right)_{0}, \ldots, \delta\left(m_{\delta}\right)_{i-1}\right\}\right) \vdash \phi\left(m_{\delta}\right)$.

By definition, $W^{s} \subseteq \Gamma\left(E^{\prime}\right)$. Furthermore, we have $\operatorname{CON}\left(\delta_{j}\right)\left(m_{\delta}\right) \in \Gamma\left(E^{\prime}\right)$ for $j<$ $i$ by the induction hypothesis. This implies that $\phi\left(m_{\delta}\right) \in \Gamma\left(E^{\prime}\right)$, because $\Gamma\left(E^{\prime}\right)$ is deductively closed.

- If $\delta_{i}=(\delta)_{a}$, then let $\phi=\operatorname{PRE}(\delta)$.

* First, consider $(\delta)_{a}^{m}$ : By Lemma 4, we have ok $\left(m_{\delta}\right) \in \Gamma\left(E^{\prime}\right)$. By Theorem 4.1 .8 of [DS00a], we conclude that $\delta_{b_{1}} \notin G D\left(D^{t}, E\right)$ and $\delta_{b_{2}} \notin$ $G D\left(D^{t}, E\right)$; therefore $\mathrm{ko}\left(m_{\delta}\right) \notin E^{\prime}$. Hence, ok $\left(n_{\delta}\right) \in \Gamma(E)$.

* Next, consider $(\delta)_{a}^{n_{\delta}}$ : Since $\Gamma\left(E^{\prime}\right)$ is deductively closed, we get $\operatorname{PRE}(\delta)\left(m_{\delta}\right) \wedge$ $\operatorname{in}\left(n_{\delta}, m_{\delta}\right) \wedge$ ok $\left(n_{\delta}\right) \in \Gamma\left(E^{\prime}\right)$. Since $\delta_{a} \in G D\left(D^{t}, E\right)$, we have $\neg J U S(\delta) \notin E$. By (54), this implies $\neg J U S(\delta)\left(m_{\emptyset}\right) \notin E^{\prime}$ and so $\neg J U S(\delta)\left(m_{\delta}\right) \notin E^{\prime}$. Hence, $\operatorname{CON}(\delta)\left(m_{\delta}\right) \wedge \operatorname{ap}\left(n_{\delta}\right) \in \Gamma(E)$.

* Finally, we have $i n\left(n_{\delta}, m_{\delta}\right) \in W^{s} \subseteq \Gamma\left(E^{\prime}\right)$ and by construction of $E^{\prime}$ that $i n\left(n_{\delta}, m_{k}\right) \notin E^{\prime}$ for $k \neq \delta$. Hence, we infer from $\frac{: \neg i n\left(n_{\delta}, m_{k}\right)}{\neg i n\left(n_{\delta}, m_{k}\right)} \in D^{s}$ that $\neg i n\left(n_{\delta}, m_{k}\right) \in \Gamma\left(E^{\prime}\right)$ for $k \neq \delta$. Since $\Gamma\left(E^{\prime}\right)$ is deductively closed and since $\Gamma\left(E^{\prime}\right)$ contains Formula (27), we deduce that ap $\left(m_{\delta}\right) \in \Gamma\left(E^{\prime}\right)$.

- If $\delta_{i}=(\delta)_{b_{1}}$, then we have $\operatorname{PRE}(\delta) \notin E$. By (54), this implies $\operatorname{PRE}(\delta)\left(m_{\emptyset}\right) \notin$ $E^{\prime}$ and so $\operatorname{PRE}(\delta)\left(m_{\delta}\right) \notin E^{\prime}$. By Lemma 4, we have ok $\left(m_{\delta}\right) \in \Gamma\left(E^{\prime}\right)$. Hence, $\mathrm{bl}\left(m_{\delta}\right) \in \Gamma(E)$.

- If $\delta_{i}=(\delta)_{b_{2}}$, then we have already shown that $\neg\left(\operatorname{CON}(\delta)\left(m_{\delta}\right) \wedge J U S(\delta)\left(m_{\delta}\right)\right) \in$ $\Gamma\left(E^{\prime}\right)$. By Lemma 4, we have ok $\left(m_{\delta}\right) \in \Gamma\left(E^{\prime}\right)$. Since $\Gamma\left(E^{\prime}\right)$ is deductively closed, we get $\neg\left(\operatorname{CON}(\delta)\left(m_{\delta}\right) \wedge J U S(\delta)\left(m_{\delta}\right)\right) \wedge$ ok $\left(n_{\delta}\right) \in \Gamma\left(E^{\prime}\right)$. Hence, bl $\left(m_{\delta}\right) \in \Gamma\left(E^{\prime}\right)$.

In all, we obtain $v \in \Gamma\left(E^{\prime}\right)$, the desired contradiction.

Since both $E^{\prime}$ and $\Gamma\left(E^{\prime}\right)$ are deductively closed, we get that $E^{\prime} \subseteq \Gamma\left(E^{\prime}\right)$.

This completes the proof showing that $E^{\prime}$ is an extension of $\mathcal{S}\left(\left(D, W,<^{\prime}\right)\right)$.

Proof 5.3 Let $(D, W)$ be a semi-normal default theory over $\mathcal{L}$ and define $<$ by: for every $\delta \in D$ let $\left\{\delta_{\downarrow}\right\}<\{\delta\}<\left\{\delta_{\uparrow}\right\}$.

if part According to Corollary 4.2 of [DS00a], we have that $E^{\prime}$ is an extension of $\mathcal{T}((D, W, \emptyset))$ iff $E^{\prime} \cap \mathcal{L}$ is an extension of $(D, W)$.

According to Theorem 5.2, if $E^{\prime}$ is an extension of $\mathcal{T}((D, W,<))$ then $E$ is an extension of $\mathcal{S}((D, W,<))$ where $E^{\prime} \cap \mathcal{L}=\downarrow(E)$. Hence, we have that $\downarrow(E)$ is an extension of $(D, W)$.

only-if part (Outline)

Given the assumptions in the statement of the theorem, we make the following simplifications to Definition 5.3. 
1. Occurrences of ko can be replaced by bl (since ko is used for readability; see also Theorem 5.1.3). Then line (26) of Definition 5.3 can be deleted.

2. $c(m, n)$ can be replaced by $\operatorname{CON}(n)(x)$ (since $c(\cdot, \cdot)$ was introduced only to avoid a space blowup in the translation).

Thus (21) can be deleted and (18) simplified.

3. The family of languages $\mathcal{L}(m)$ for $m \in M$ can be replaced by the language $\mathcal{L}$. That is, formulas don't need to be indexed by the set in which they appear since now all results (i.e. consequents of rule applications) are propagated to all sets (with the exception of the trivial set $\left.\left\{\delta_{\uparrow}\right\}\right)$ via (20) and (28). So the added argument to formulas can be dropped, as can (20) and (28).

4. We can unambiguously use the name $n$ to refer to both a default, and the set containing that default.

5. ap $(y \cdot x)$ can be replaced by ap $(x)($ since ap $(x)$ can be derived only via ap $(y \cdot x)$ using (27)).

As well, (27) can be deleted.

6. Names in $M$ are redundant. Consequently, occurrences of in can be deleted. As well, the second default in (19) can be deleted, as can (22).

This leaves the following simplified translation for Definition 5.3.

$$
\begin{aligned}
D_{N} & =\left\{\frac{\alpha \wedge \mathrm{ok}(n \cdot n): \beta}{\gamma \wedge \mathrm{ap}(n)} \mid n: \frac{\alpha: \beta}{\gamma} \in D\right\} \\
D_{M} & =\left\{\frac{\mathrm{ok}(n): \neg \mathrm{bl}(n)}{\mathrm{ok}(n \cdot n)} \mid n: \frac{\alpha: \beta}{\gamma} \in D\right\} \\
& \cup\left\{\frac{\mathrm{ok}(n): \neg \alpha}{\mathrm{bl}(n)} \mid n: \frac{\alpha: \beta}{\gamma} \in D\right\} \\
& \cup\left\{\frac{\neg \beta \wedge \mathrm{ok}(n):}{\mathrm{bl}(n)} \mid n: \frac{\alpha: \beta}{\gamma} \in D\right\} \\
D_{\neg} & =\left\{\frac{: \neg(x \prec y)}{\neg(x \prec y)}\right\} \\
W_{\prec} & =\left\{\text { ok }\left(m_{D}\right)\right\} \\
& \cup\left\{n_{i} \prec n_{j} \mid\left(\left\{\delta_{i}\right\},\left\{\delta_{j}\right\}\right) \in<\right\} \\
& \cup\{\forall x \in N .[\forall y \in N .(x \prec y) \supset(\mathrm{bl}(y) \vee \operatorname{ap}(y))] \supset \text { ok }(n)\}
\end{aligned}
$$

But this is just the translation $\mathcal{T}((D, W, \emptyset))$ of [DS00a] except that the rule

$$
\frac{\alpha \wedge \mathrm{ok}(n): \beta}{\gamma \wedge \operatorname{ap}(n)}
$$

in [DSO0a] is replaced by the pair of rules

$$
\frac{\alpha \wedge \mathrm{ok}(n \cdot n): \beta}{\gamma \wedge \mathrm{ap}(n)}, \quad \frac{\operatorname{ok}(n): \neg \mathrm{bl}(n)}{\operatorname{ok}(n \cdot n)}
$$


in $D_{N}$ and the first rule in $D_{M}$ above. However, modulo the language $\mathcal{L}$, the pair of rules above can be shown to have the same effect as the rule from [DS00a].

Thus, under the premisses of the theorem, Definition 5.3 is essentially the same as the translation $\mathcal{T}((D, W, \emptyset))$. From Corollary 4.2 of [DS00a], we have that $E^{\prime}$ is an extension of $\mathcal{T}((D, W, \emptyset))$ iff $E^{\prime} \cap \mathcal{L}$ is an extension of $(D, W)$, from which our result obtains.

\section{Proofs for Section 6}

Proofs for this section are found in [DS02].

\section{References}

[BE00] G. Brewka and T. Eiter. Prioritizing default logic. In St. Hölldobler, editor, Intellectics and Computational Logic - Papers in Honour of Wolfgang Bibel, pages 27-45. Kluwer Academic Publishers, 2000.

[BEFK99] R. Ben-Eliyahu, N. Francez, and M. Kaminski. Similarity preservation in default logic. Annals of Mathematics and Artificial Intelligence, 25(1/2):137-160, 1999.

[BG94] G. Brewka and T. Gordon. How to buy a porsche: An approach to defeasible decision making. In AAAI-94 Workshop on Computational Dialectics, pages 28-38, Seattle, WA, July 1994. AAAI Press.

[BH93] F. Baader and B. Hollunder. How to prefer more specific defaults in terminological default logic. In R. Bajcsy, editor, Proceedings of the International Joint Conference on Artificial Intelligence, pages 669-674. Morgan Kaufmann Publishers, 1993.

[Bre94a] G. Brewka. Adding priorities and specificity to default logic. In L. Pereira and D. Pearce, editors, European Workshop on Logics in Artificial Intelligence (JELIA'94), Lecture Notes in Artificial Intelligence, pages 247-260. Springer Verlag, 1994.

[Bre94b] G. Brewka. Reasoning about priorities in default logic. In Proceedings of the AAAI National Conference on Artificial Intelligence, volume 2, pages 940-945. AAAI Press/The MIT Press, 1994.

[CMT96] P. Cholewiński, V. Marek, and M. Truszczyński. Default reasoning system DeReS. In Proceedings of the Fifth International Conference on the Principles of Knowledge Representation and Reasoning, pages 518-528. Morgan Kaufmann Publishers, 1996.

[Dix92] J. Dix. On cumulativity in default logic and its relation to Poole's approach. In B. Neumann, editor, Proceedings of the European Conference on Artificial Intelligence, pages 289-293. John Wiley \& sons, 1992.

[DS00a] J. Delgrande and T. Schaub. Expressing preferences in default logic. Artificial Intelligence, 123(1-2):41-87, 2000. 
[DS00b] J.P. Delgrande and T. Schaub. The role of default logic in knowledge representation. In J. Minker, editor, Logic-Based Artificial Intelligence, pages 107-126. Kluwer Academic Publishers, 2000.

[DS02] J.P. Delgrande and T. Schaub. Reasoning credulously and skeptically within a single extension. Journal of Applied Non-Classical Logics, 2002. to appear.

[ELM ${ }^{+97]}$ T. Eiter, N. Leone, C. Mateis, G. Pfeifer, and F. Scarcello. A deductive system for nonmonotonic reasoning. In J. Dix, U. Furbach, and A. Nerode, editors, Proceedings of the Fourth International Conference on Logic Programming and Non-Monotonic Reasoning, volume 1265 of Lecture Notes in Artificial Intelligence, pages 363-374. Springer-Verlag, 1997.

[GS97] M. Gelfond and T. Son. Reasoning with prioritized defaults. In J. Dix, L. Pereira, and T. Przymusinski, editors, Third International Workshop on Logic Programming and Knowledge Representation, volume 1471 of Lecture Notes in Computer Science, pages 164-223. Springer-Verlag, 1997.

[Jan99] T. Janhunen. Classifying semi-normal default logic on the basis of its expressive power. In M. Gelfond, N. Leone, and G. Pfeifer, editors, Proceedings of the Fifth International Conference on Logic Programming and Nonmonotonic Reasoning (LPNMR'99), volume 1730 of Lecture Notes in Artificial Intelligence, pages 19-33. Springer Verlag, 1999.

[McC86] J. McCarthy. Applications of circumscription to formalizing common-sense knowledge. Artificial Intelligence, 28:89-116, 1986.

[Moo85] R.C. Moore. Semantical considerations on nonmonotonic logic. Artificial Intelligence, 25:75-94, 1985.

[NS97] I. Niemelä and P. Simons. Smodels: An implementation of the stable model and wellfounded semantics for normal logic programs. In J. Dix, U. Furbach, and A. Nerode, editors, Proceedings of the Fourth International Conference on Logic Programming and Nonmonotonic Reasoning, pages 420-429. Springer-Verlag, 1997.

[Poo88] D.L. Poole. A logical framework for default reasoning. Artificial Intelligence, 36(1):27-48, 1988.

[Rei80] R. Reiter. A logic for default reasoning. Artificial Intelligence, 13(1-2):81-132, 1980.

[Rei87] R. Reiter. A theory of diagnosis from first principles. Artificial Intelligence, 32(1):5796, 1987.

[Rin95] J. Rintanen. On specificity in default logic. In Proceedings of the International Joint Conference on Artificial Intelligence, pages 1474-1479, Montreal, 1995.

[Thi01] M. Thielscher. The qualification problem: A solution to the problem of anomalous models. Artificial Intelligence, 121(1-2):1-37, 2001. 